\title{
Complexity Behaviors of Volatility Dynamics for Stochastic Potts Financial Model
}

Jie Wang ( $\square$ jiewang@ncut.edu.cn )

North China University of Technology

\section{Research Article}

Keywords: Stochastic Potts dynamics, volatility statistic, permutation Lempel-Ziv complexity, multi-scale fractional sample entropy, volatility duration average intensity

Posted Date: March 16th, 2021

DOI: https://doi.org/10.21203/rs.3.rs-268116/v1

License: (c) (i) This work is licensed under a Creative Commons Attribution 4.0 International License. Read Full License 


\title{
Complexity behaviors of volatility dynamics for stochastic Potts financial model
}

\author{
Jie $\operatorname{Wang}^{a, 1}$ \\ ${ }^{a}$ Department of Statistics, College of Science \\ North China University of Technology \\ Beijing 100144, P.R. CHINA
}

\begin{abstract}
To investigate the price fluctuation mechanism of stock markets, this research aims to develop a novel stochastic financial model based on Potts dynamics and compound Poisson process. The new model considers two aspects: information interaction among traders and the uncertain events outside the system. Then, three different volatility statistics (return series $r_{t}$, absolute return series $\left|r_{t}\right|$ and volatility duration average intensity $V_{t}$ ) are introduced to explore the volatility and complexity properties of the proposed model. The descriptive statistical methods, power-law analysis are studied to validate the practicable of the proposed stochastic financial model. The Permutation LempelZiv complexity of moving average series are referred to different volatility sequences to evaluate the complexity of the simulative data from proposed model and the real data from stock market. Moreover, the complexity analysis of Fractional sample entropy and Multiscale fractional sample entropy method are improved to illustrate the complexity of volatility behaviors in different scales. Compared with the real stock data, the empirical results demonstrate that the new model could reproduce the fluctuation and volatility
\end{abstract}

\footnotetext{
${ }^{1}$ Corresponding author. Tel.: +86 10 88803272. Email: jiewang@ncut.edu.cn.
} 
behaviors of real stock markets to some extent.

Keywords Stochastic Potts dynamics; volatility statistic; permutation Lempel-Ziv complexity; multi-scale fractional sample entropy; volatility duration average intensity 


\section{Introduction}

Recently, nonlinear fluctuation and volatility dynamics of financial markets has been a key problems, because its corresponding financial time series are time-varying, non-linear, and chaotic. Financial market is regarded as a complex dynamic system, then rehabilitating and investigating nonlinear complexity characteristics of financial data have been an active topic for deeply comprehension and management of stock markets $[1,2,3,4,5]$. Recently, theoretical and practical studies show that price variations exists significant statistical properties, such as power-law behavior, fat-tailed distribution, multifractality and complexity dynamics, etc. $[6,7,8,9,10,11]$. Numerous financial price dynamics models have been effectively constructed in simulating the volatility complex behaviors of financial markets based on statistical physics systems and stochastic particle systems $[12,13,14,15,16,17,18,19,20,21,22]$. Lux[12] provided a stochastic framework of sentiment dynamics and explored the added explanatory power of nonlinearities introduced through the interaction of agents. Boswijk et al.[13] estimated a dynamic asset pricing model characterized by heterogeneous boundedly rational agents. Mike and Farmer[16] developed a behavioral model for liquidity and volatility based on empirical regularities in trading order flow in the London Stock Exchange. Stauffer and Penna[17] established price models using percolation system in which a percolation cluster interpreted a group of traders who held the same trading attitude. Wang and Wang[18, 21] proposed a random financial time series model of the attitudes of market traders based on Potts dynamic system. Wang et al.[19] improved a novel nonlinear random interacting price dynamics by the combination of lattice oriented percolation and Potts dynamics, 
which concerns with the instinctive random fluctuation and the fluctuation caused by the spread of the investors' trading attitudes, respectively. Zhang and Wang[20], Bornholdt and Wagner[22] used the Ising model to construct a financial price model, where the essential trading framework can be recast into an Ising-type spin model. The practical consequences reveal that these simulative models could reflect the statistical characteristics of real financial systems.

Price fluctuation dynamic and volatility dynamic are important factors in the research of financial dynamic system. Security logarithmic return is usually selected to discuss the fluctuation statistical characteristics. A variety of statistics for the measurement of price volatility behaviors, such as return interval[18, 23], volatility duration[24], continuous fluctuation intensity[25], maximum change rate series and average change rate series[26]. Wang et al.[23] studied the return intervals between price volatilities, and found that the probability density function of return intervals follows a scaling function. Yang et al.[24] used the volatility duration to study the relationship between the duration and intensity relationship of financial time series. Wang and Li et al. [25, 26] defined different intensity volatility statistics based on the volatility duration method. In this work, a novel volatility component named volatility duration average intensity is developed on the basis of volatility duration and the average change rate series, which can provide a new method on the statistical characteristics of financial market fluctuations. In addition, the research on the complexity analysis of financial system has drawn wide attention, which helps investors to better understand the variation statistics properties during investment decision. Many information theory methods to estimate the complexity has been used 
to different dynamic systems, such as multiscale sample entropy [27, 28], Lempel-Ziv complexity[29, 30, 31], weighted fractional permutation entropy and fractional sample entropy[32], etc. Particularly, LZC put forward by Lempel and Ziv [33] and its derivatives have found numerous applications in characterizing the randomness of finite data-series by measuring the number of distinct sub-strings.

Inspired by the above research results, we establish a new stochastic financial dynamic model based on Potts system and random theory. Potts system is a famous statistical physics approach to modeling non-equilibrium statistical mechanics and is an extension of the Ising model[34, 35, 36]. Since real stock markets sometimes appears extreme jump volatilities, which supports intensive and notable impacts on price changes, introducing random jumps to model stock market fluctuations is feasible in studying phenomenon of stock markets such as option pricing and risk management[37]. To compare with the fluctuation and volatility dynamics of price model and real stock market, the logarithmic return series $r_{t}$, absolute return series $\left|r_{t}\right|$ and volatility duration average intensity series $V_{t}$ of the simulative data from the proposed model with different model parameters $\beta, \lambda$ and four international stock indexes - Shanghai Stock Exchange Composite Index (SSE), Hang Seng Index (HSI), Nikkei 225 (N225) and Dow Jones Industrial Average (DJIA) are investigated. The specific volatility analysis methods mainly includes two aspects, the statistical characteristics analysis and the complexity behavior analysis. We first analyze the descriptive statistics, fat-tail phenomena and power-law behaviors of volatility sequences for simulative data and real stock data. Then, the complexity behaviors of $r_{t},\left|r_{t}\right|$ and $V_{t}$ are followed up with permutation Lempel-Ziv complexity (PLZC) method, which is a fresh 
complexity measure utilized to inspect the relevant uncertainty and complexity behaviors for financial time series $[26,31,38]$. In addition, the fractional sample entropy (FSE)[32] and the advanced method multiscale fractional sample entropy (MFSE) are adopted as complexity estimations for simulative data and real data. As has been stated, there are three main contributions in this paper. The first is to provide a novel financial price modeling process, in which the information interaction mechanism of market traders and extreme jump volatilities is mimicked by the Potts dynamic system with random jump. The second is to study the statistical and complexity behaviors of simulative time series with three different volatility sequences. Thirdly, a fresh complexity estimation method are improved based on FSE analysis, called MFSE. By applying PLZC, FSE and MFSE, the complexity of the fluctuation and volatility components are researched for simulative data and the real data. We hope the research results of this article can provide valuable opinions for the modeling and complexity analysis of financial market.

\section{Stochastic Potts financial model}

\subsection{Brief description of Potts model}

Potts system is a famous statistical physics dynamics $[34,35,36]$ proposed by Potts in the early 1950s. It is an promotion system of the Ising model, which considers more than two components in the mechanism. The Potts model is related to a number of outstanding problems in lattice statistics, and its critical behavior is more abundant and more generalized than that of the Ising system. Considering he two-dimensional integer lattice $\mathbb{Z}^{2}$ and denoting by $\mathfrak{B}$ the set of bonds of the lattice (pairs of nearest neighbors). In 
the $Q$-state Potts model, let $\Omega_{\mathbb{Z}^{2}}=\{1,2, \cdots, Q\}^{\mathbb{Z}^{2}}$ denote the space of spin configurations on $\mathbb{Z}^{2}$, an element of $\Omega_{\mathbb{Z}^{2}}$ usually notated $\sigma=\left\{\sigma_{i}: i \in \mathbb{Z}^{2}\right\}$. The spin $\sigma_{i}$ take on oneinteger values from 1 to $Q$, and the $Q$ is a parameter of the model. For every $\sigma \in \Omega_{\mathbb{Z}^{2}}$, the Hamiltonian system of the $Q$-state Potts model $(J>0)$ is

$$
H_{\mathbb{Z}^{2}, b}(\sigma)=-J \sum_{<i, j>} \delta_{\sigma_{i}, \sigma_{j}}-b \sum_{i} \delta_{\sigma_{i, 1}}
$$

where $\delta$ is the Kroeneker symbol, $\delta_{\sigma_{i}, \sigma_{j}}=1$ only when $\sigma_{i}=\sigma_{j},\langle i, j>$ denotes pairs of nearest-neighbor spins on the lattice, and the applied magnetic field $b$ acts on the (arbitrarily chosen) state 1 . Then the partition function is

$$
Z_{\mathbb{Z}^{2}, h}(\sigma)=\sum \exp \left(K \sum_{<i, j>} \delta_{\sigma_{i}, \sigma_{j}}+h \sum_{i} \delta_{\sigma_{i, 1}}\right)
$$

where $K=\beta J$ and $h=\beta b, \beta=1 /\left(k_{B} T\right), k_{B}$ is the Boltzmann constant and $T$ is the temperature. Here the $Q$-state Potts model with no external magnetic field $(b=0$ and $h=0)$ is studied. The model stands an order-disorder transformation when $d \geq 2$, and the critical value is $\beta_{c}=\ln (1+\sqrt{Q})$ in $d=2$. When $\beta>\beta_{c}$ the $Q$-fold permutation symmetry of Eq. (1) is broken, and one of the $Q$ different ground states is picked over.

\subsection{Modelling Stochastic Potts financial dynamics}

We here establish a new Stochastic financial model by integrating the two-dimensional 3 -state Potts dynamics and compound Poisson process with random jumps on a $L \times$ $L$ lattice. The proposed model premeditates the fluctuation caused by the interaction intensity among investors and the inherent randomness of the system, respectively. In the fist part, the intensity of interactivity between adjoining components is quite fundamental. 
It changes on the basis of their position in the lattice and, as conventional of Potts models, little changes in interaction regulations do not alter the group attributes. Although the optimal number of states existed in Potts model is thus not restricted to 3, we apply a 3-state Potts model that simulates (i) traders with the selling decision, (ii) traders with the buying decision, and (iii) traders with no trading decision, which we categorize as type 1 , type 2 , and type 3 , respectively. Consider that financial volatility behavior is highly influenced by the quantity of traders $\omega^{(1)}(t)$ (traders of type 1$), \omega^{(2)}(t)$ (traders of type 2 ), and $\omega^{(3)}(t)$ (traders of type 3 ). We asses a single stock and suppose that there are $L^{2}$ traders of this stock who are situated in a square-lattice $L \times L \subset \mathbb{Z}^{2}$, and each trader can deal a unit number of stock at each time $t \in\{1,2, \cdots, T\}$. At this time, the fluctuation of stock price is extremely affected by the number of traders who take buying or selling locations. When the number of traders in selling locations is smaller than the number of traders in buying locations, the market participants believe that the stock price is undervalued, then the stock price gradually increases. The similar is correct in the opposite situation. Let $\omega_{i j}$ be the trading location of a trader $(1 \leq i \leq L, 1 \leq j \leq L)$ at time $t$, and $\omega(t)=\left(\omega_{11}(t), \cdots, \omega_{1 L}(t), \cdots, \omega_{L 1}(t), \cdots, \omega_{L L}(t)\right)$ be the configuration of locations for $L^{2}$ traders. A space of all configurations of locations for $L^{2}$ traders from time 1 to $t$ is defined by $\mathcal{W}=\{\omega: \omega=(\omega(1), \cdots, \omega(t))\}$. For a given configuration $\omega \in \mathcal{W}$ and a trading day $t$, let $M^{(k)}(\omega(t))=\left|\omega^{(k)}(t)\right|(k=1,2,3)$, which represents the number of $\omega^{(1)}(t), \omega^{(2)}(t)$, and $\omega^{(3)}(t)$ at time $t$, respectively. Suppose that the price changes are in accordance with the difference between demand and supply $M^{(k)}(\omega(t))$ $(k=1,2,3)$, which is controlled by the strength parameter $\beta$, where $\beta$ stands for the 
intensity of information dissemination. Then define a random variable $\xi_{t}$ with values 1 , $-1,0$ when an trader is buying, selling or neutral with probabilities $p_{1}, p_{-1}$ or $1-\left(p_{1}+p_{-1}\right)$, respectively. Meanwhile these traders send a bullish, bearish, or neutral message into the market. From the above depiction and $[39,40]$, the stock price at trading day $t$ is given as

$$
\begin{gathered}
\mathcal{P}^{1}(t)=\exp \left\{\alpha_{1} \sum_{k=1}^{3} \frac{\mathcal{M}^{(k)}(t)}{L^{2}}\right\} \mathcal{P}^{1}(t-1) \\
\mathcal{M}^{(k)}(t)=M^{(k)}(\omega(t)) \times \gamma_{k} \times \xi_{t}^{k},
\end{gathered}
$$

where $\alpha_{1}(>0)$ is the depth indicator of the market and $\gamma_{k}$ is the effective strength of trading attitudes in the stock market such that $\gamma_{1}+\gamma_{2}+\gamma_{3}=1$. Then we have

$$
\mathcal{P}^{1}(t)=\mathcal{P}(0) \exp \left\{\alpha_{1} \sum_{s=1}^{t} \sum_{k=1}^{3} \frac{\mathcal{M}^{(k)}(s)}{L^{2}}\right\}
$$

where $\mathcal{P}(0)$ is the stock price at time 0 .

Next, we discuss the sharp fluctuations of the financial market from the inherent randomness of the system. Let $\mathcal{B}\left(\zeta_{s}\right)$ be the $s$-th price jump, which is an independent identical distributed (i.i.d.) series, and suppose that $\eta_{t}$ is a Poisson process with intensity $\lambda$. The two stochastic processes $\mathcal{B}\left(\zeta_{s}\right)$ and $\eta_{t}$ are independent of each other. Then the random sharp fluctuations for the financial system is described as

$$
\mathcal{P}^{j u m p}(t)=\mathcal{P}(0) \exp \left\{\alpha_{2} \sum_{s=1}^{\eta_{t}} \mathcal{B}\left(\zeta_{s}\right)\right\},
$$

where $\alpha_{2}(>0)$ is the sensitivity parameter of the market.

In the final stage of the modelling, we assume the market volatility formation mechanism is caused by two parts. The first part is the interaction behaviors among market 
traders is explained by Potts dynamics. The second part is the randomness of the sharp fluctuations which is expressed by compound Poisson process with random jumps. Combining the above two price models, the stock price process on the $t$-th trading day is defined as

$$
\mathcal{P}(t)=\mathcal{P}(0) \exp \left\{\alpha_{1} \sum_{s=1}^{t} \sum_{k=1}^{3} \frac{\mathcal{M}^{(k)}(s)}{L^{2}}+\alpha_{2} \sum_{s=1}^{\eta_{t}} \mathcal{B}\left(\zeta_{s}\right)\right\},
$$

where $\mathcal{P}(0)$ is the stock price at time 0 . The corresponding formula of the stock logarithmic return is

$$
r(t)=r_{t}=\ln \mathcal{P}(t)-\ln \mathcal{P}(t-1), \quad t \in\{1,2, \cdots, T\}
$$

\section{Data description and statistical properties}

\subsection{Data preparation and processing}

To investigate the complexity behavior of financial price fluctuations, we choose daily closing data of four real global indexes: Shanghai Stock Exchange Composite Index (SSE), Hang Seng Index (HSI), Nikkei 225 (N225) and Dow Jones Industrial Average (DJIA) from Jan 3, 2011 to Jan 4, 2021. The simulated data derived from the proposed stochastic Potts financial model with parameters $\beta=\beta_{c}$ and $\beta=3$ for Potts system, parameters $\lambda=5$ and $\lambda=10$ for the compound Poisson jump and the number of trading days $T$ is set to 2500 . It is worth mentioning that the selection of model parameters is not fixed, where different values should be chosen due to multiple simulative experiments for different markets. Fig. 1 displays the price series and the corresponding returns for the real data and the simulated data. 


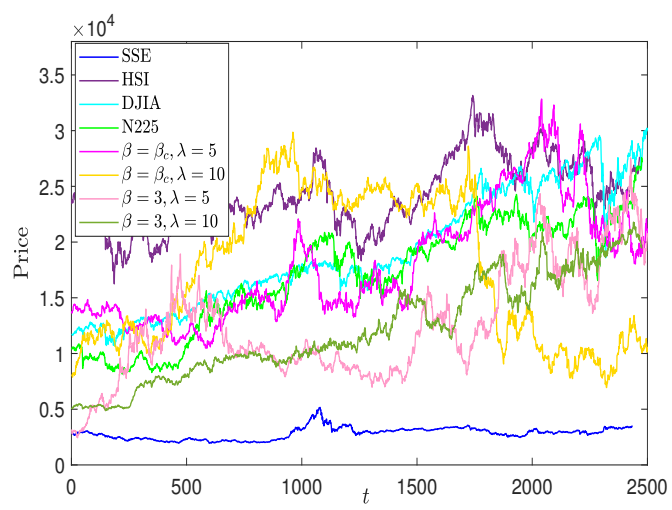

(a)

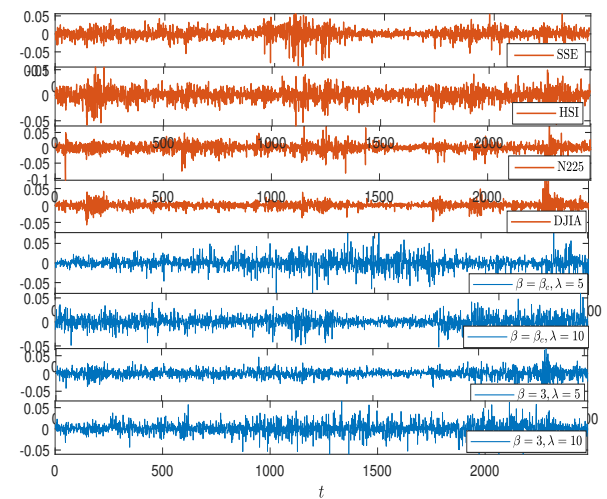

(b)

Figure 1: (a) Price time series of real indexes and the simulative data of the stochastic Potts financial model. (b) Plots of return series $r_{t}$ for real indexes and the simulative data of the stochastic Potts financial model.

In order to study the statistical and complexity behaviors of volatility sequences, we analyze the logarithmic returns $r_{t}$, absolute returns sequences $\left|r_{t}\right|$ and a new return volatility component, volatility duration average intensity $V_{t}$ for the daily price changes. The daily logarithmic return series $r_{t}$ is the most commonly used statistic of fluctuations analysis in financial theory, which can directly express the price changes between two consecutive trading days. $\left|r_{t}\right|$ can be viewed as corresponding volatility sequences. $V_{t}$ is a new method to estimate the volatility of stock prices, which is an improved form of the volatility duration and the average variation intensity of volatility. In the following, we here introduce the volatility duration length series $I(t)$ at trading day $t$. If $\left|r_{t+1}\right|>\left|r_{t}\right|$, indicates that there is a local upward movement at trading day $t$. If $\left|r_{t+1}\right|<\left|r_{t}\right|$, the volatility sequences shows sectional downward trend at day $t . I(t)$ is defined to record 


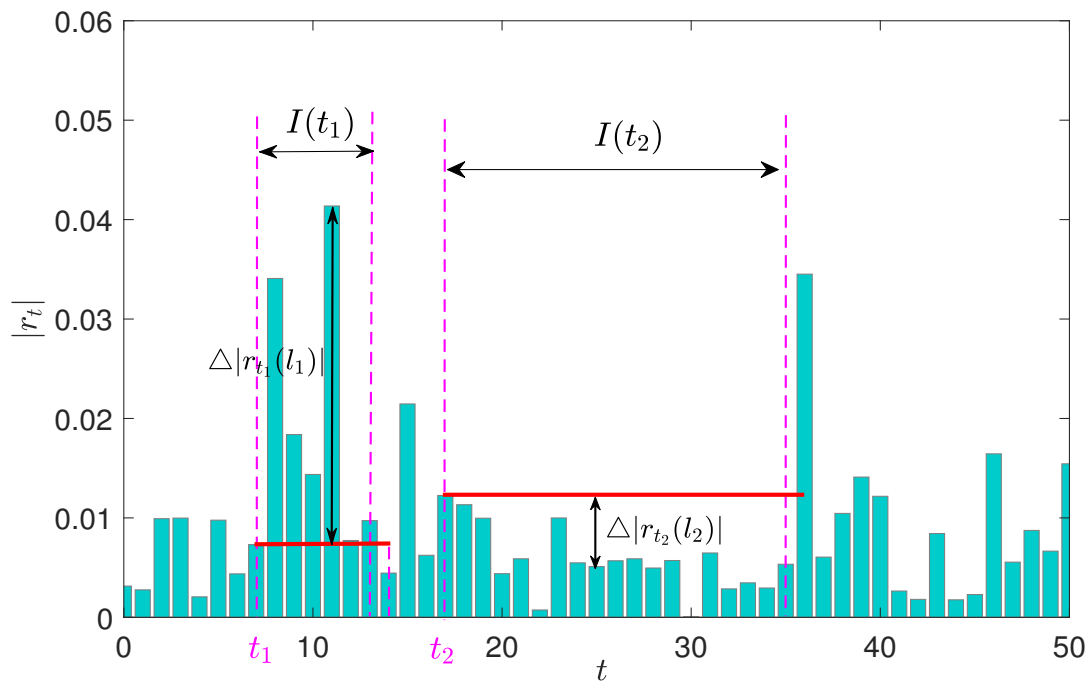

Figure 2: Illustrations of $I(t), \Delta\left|r_{t}(i)\right|$ in the volatility series.

the local trend length of duration volatility intensity, which is descried as

$$
I(t)=\left\{\begin{array}{l}
\max \left\{\tau:\left|r_{t+i}\right|>\left|r_{t}\right|, \quad i=1,2, \ldots, \tau\right\} \\
i f\left|r_{t+i}\right|-\left|r_{t}\right|>0, \\
\max \left\{\tau:\left|r_{t+i}\right|<\left|r_{t}\right|, \quad i=1,2, \ldots, \tau\right\} \\
i f\left|r_{t+i}\right|-\left|r_{t}\right|<0 .
\end{array}\right.
$$

In the case of $\left|r_{t+1}\right|=\left|r_{t}\right|, I(t)=0$. Then, let $\Delta\left|r_{t}(i)\right|$ denote the volatility difference module at time $i$ in the duration $I(t)$ at time $t$, which is described as

$$
\Delta\left|r_{t}(i)\right|=|| r_{t}(t+i)|-| r_{t}(i)||, \quad 1 \leq i \leq I(t)
$$

The average variation intensity of volatility $A_{t}$ in the volatility duration series $I(t)$ is defined as

$$
A_{t}=\frac{1}{I(t)} \sum_{i}^{I(t)} \Delta\left|r_{t}(i)\right|, \quad t=1,2, \ldots, N-1
$$




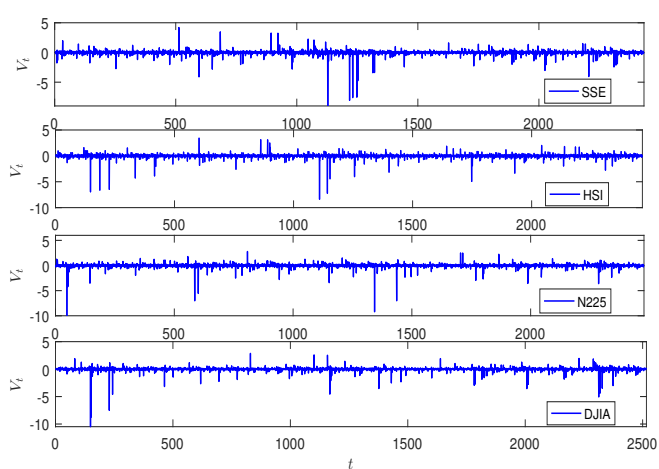

(a)

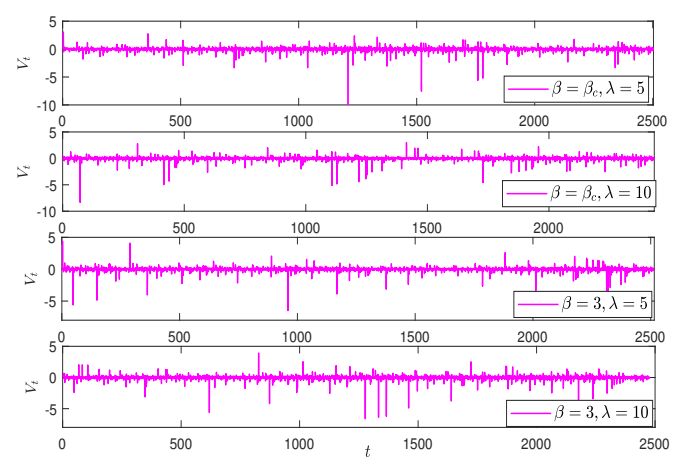

(b)

Figure 3: (a) The volatility duration average intensity sequences $V_{t}$ of real data for different market indexes. (b) The volatility duration average intensity sequences $V_{t}$ sequences of the simulative data with $\left\{\beta=\beta_{c}, \lambda=5\right\},\left\{\beta=\beta_{c}, \lambda=10\right\},\{\beta=3, \lambda=5\},\{\beta=3, \lambda=$ $10\}$ for the stochastic Potts financial model.

The volatility duration average intensity $V_{t}, t=1,2, \ldots, N-1$ based on series $I(t)$ and $A_{t}$ is defined as

$$
\begin{aligned}
V_{t} & =\operatorname{sign}\left(\left|r_{t+1}\right|-\left|r_{t}\right|\right) \times\left(I(t) \times A_{t}\right)^{p} \\
& =\operatorname{sign}\left(\left|r_{t+1}\right|-\left|r_{t}\right|\right) \times\left(\sum_{i}^{I(t)} \Delta \mid r_{t}(i)\right)^{p}
\end{aligned}
$$

where parameter $p$ can adjust extreme values. We set $p=1 / 2$ in this paper. Fig. 2 shows an example of volatility duration $I(t)$ and volatility difference component $\Delta\left|r_{t}(i)\right|$ in $V_{t}$. The final $V_{t}$ sequences of real data for different stock markets and the simulative data for proposed model are demonstrated in Fig.3.

\subsection{Statistical properties}

The statistical properties of financial fluctuation and volatility series has always been 
the key basis of the theory and practical in asset portfolio, asset pricing, financial risk management, stock volatility forecasting, etc. [39, 41, 42, 43]. Empirical results have indicated that the probability density of returns are not follow Gaussian distribution, but demonstrates the significant features of excess kurtosis and fat tail. Actually, the logarithmic returns obeys a power-law distribution, $P\left(\left|r_{t}\right|>x\right) \sim x^{-\nu}$, where $r_{t}$ is the logarithmic return of price series and the exponent of $\nu$ is approximately equal to $3[44]$. The corresponding descriptive statistics of these different return series and volatility duration average intensity sequences illustrated in Table 1 . Through the statistical research, the values for return series are between $(-0.15,0.15)$, The mean values are basically around 0 , the values of kurtosis are bigger than the value of standard normal distribution 3 and the corresponding skewness values are inconsistent with 0 of the Gaussian distribution. For sequences $V_{t}$, the specific values are between $(-15,5)$, the standard deviation values are extremely approaching, the value of kurtosis are bigger than 3 and skewness are nonzero values. It evidences that the simulative data and the real data have excess kurtosis and nonzero skewness for return series and volatility duration average intensity sequences. The distributions of return series and volatility duration average intensity sequences are different from the Gaussian distribution. In addition, the exponent of $\nu$ for returns series all are approximately equal to 3, and for $V_{t}$ sequence the values of $\nu$ are close to 1.6. Figure 4(a)(b) exhibit the probability distributions of the $r_{t}$ and $V_{t}$ sequences for the simulative data, the real data and the Gaussian distribution, where the simulative data are with parameters $\left\{\beta=\beta_{c}, \lambda=5\right\},\left\{\beta=\beta_{c}, \lambda=10\right\},\{\beta=3, \lambda=5\},\{\beta=3, \lambda=10\}$, the real data are SSE, HSI, N225 and DJIA. Figure 4(c)(d) depict the power-law distributions 
Table 1: Descriptive statistics and Kolmogorov-Smirnov test for returns and $V_{t}$ series of simulation data and real data.

\begin{tabular}{|c|c|c|c|c|c|c|c|c|c|c|}
\hline \multirow[b]{2}{*}{ Returns } & \multicolumn{7}{|c|}{ Descriptive statistics } & \multicolumn{3}{|c|}{ Kolmogorov-Smirnov test } \\
\hline & Min & Max & Mean & Std & Kurtosis & Skewness & $\nu$ & $\mathrm{H}$ & CV & K-S stat \\
\hline SSE & -0.0887 & 0.0560 & $9.0996 \times 10^{-5}$ & 0.0135 & 9.6991 & -0.9574 & 2.6809 & 1 & 0.0275 & 0.4788 \\
\hline HSI & -0.0602 & 0.0552 & $7.0010 \times 10^{-5}$ & 0.0118 & 5.6250 & -0.3366 & 3.3788 & 1 & 0.0273 & 0.4819 \\
\hline $\mathrm{N} 225$ & -0.1059 & 0.0773 & $3.8861 \times 10^{-4}$ & 0.0133 & 8.7601 & -0.4524 & 3.0038 & 1 & 0.0272 & 0.4792 \\
\hline DJIA & -0.1384 & 0.1076 & $3.8158 \times 10^{-4}$ & 0.0111 & 26.2062 & -1.0124 & 2.4492 & 1 & 0.0270 & 0.4811 \\
\hline$\beta=\beta_{c}, \lambda=5$ & -0.0910 & 0.0792 & $-2.7427 \times 10^{-4}$ & 0.0137 & 8.3853 & -0.0516 & 3.0174 & 1 & 0.0271 & 0.4767 \\
\hline$\beta=\beta_{c}, \lambda=10$ & -0.0575 & 0.0594 & $1.2529 \times 10^{-4}$ & 0.0114 & 5.8287 & -0.3582 & 3.3935 & 1 & 0.0275 & 0.4827 \\
\hline$\beta=3, \lambda=5$ & -0.0899 & 0.0894 & $7.0701 \times 10^{-5}$ & 0.0109 & 10.4073 & -0.3832 & 3.2587 & 1 & 0.0270 & 0.4840 \\
\hline$\beta=3, \lambda=10$ & -0.0704 & 0.0658 & $5.4794 \times 10^{-4}$ & 0.0133 & 6.3236 & -0.1251 & 3.3717 & 1 & 0.0272 & 0.4786 \\
\hline$V_{t}$ & Min & $\operatorname{Max}$ & Mean & Std & Kurtosis & Skewness & $\nu$ & $\mathrm{H}$ & $\mathrm{CV}$ & K-S stat \\
\hline SSE & -10.2538 & 4.1989 & -0.0298 & 0.5722 & 115.0238 & -7.4699 & 1.5338 & 1 & 0.0275 & 0.3022 \\
\hline HSI & -8.3644 & 3.3896 & -0.0267 & 0.5189 & 89.7396 & -6.4875 & 1.6376 & 1 & 0.0273 & 0.3023 \\
\hline $\mathrm{N} 225$ & -15.3126 & 2.7794 & -0.0310 & 0.5902 & 231.3262 & -11.1066 & 1.6884 & 1 & 0.0272 & 0.2958 \\
\hline DJIA & -10.4831 & 2.8552 & -0.0323 & 0.5096 & 141.7198 & -8.6897 & 1.5085 & 1 & 0.0270 & 0.3216 \\
\hline$\beta=\beta_{c}, \lambda=5$ & -10.2509 & 2.8552 & -0.0255 & 0.4894 & 119.0151 & -6.9690 & 1.7480 & 1 & 0.0271 & 0.2970 \\
\hline$\beta=\beta_{c}, \lambda=10$ & -8.3276 & 2.9808 & -0.0278 & 0.4774 & 70.5508 & -5.3898 & 1.5257 & 1 & 0.0275 & 0.3070 \\
\hline$\beta=3, \lambda=5$ & -6.8464 & 4.3942 & -0.0195 & 0.4364 & 61.8496 & -3.8948 & 1.6454 & 1 & 0.0270 & 0.3093 \\
\hline$\beta=3, \lambda=10$ & -6.6106 & 3.9170 & -0.0233 & 0.4831 & 62.6157 & -4.7977 & 1.6531 & 1 & 0.0272 & 0.2987 \\
\hline
\end{tabular}

and the cumulative distributions of $\left|r_{t}\right|$ and $\left|V_{t}\right|$ for real data and the simulative data.

Moreover, single-sample Kolmogorov-Smirnov (K-S) test of the real market data and the simulative data are investigated[45]. In K-S test, the significance level in the paper is set to $5 \%$. The logical value $H=1$ is returned in the $\mathrm{K}-\mathrm{S}$ test if it rejects the null hypothesis that the distribution of normalized $r_{t}\left(V_{t}\right)$ series follows the distribution of Gaussian at the given significance level, while $H=0$ if it cannot. The empirical results are illustrated in Table 1, where 'CV' represents the critical value of of whether to accept the hypothesis and 'K-S stat' is the value of the test statistics. It is observed that all the $H$ values are 1 and 'K-S stat' values are greater than the corresponding 'CV' for all $r_{t}$ and $V_{t}$ series, thus the hypothesis is refused that the logarithmic $r_{t}$ and $V_{t}$ series distributions of simulative data and real data follow the Gaussian distribution. 


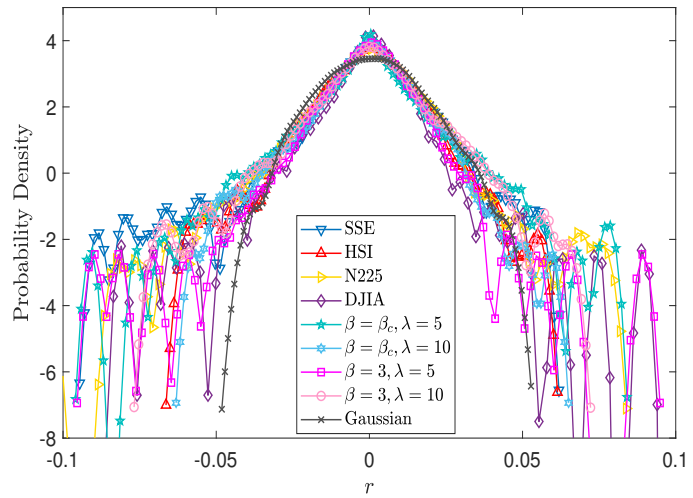

(a)

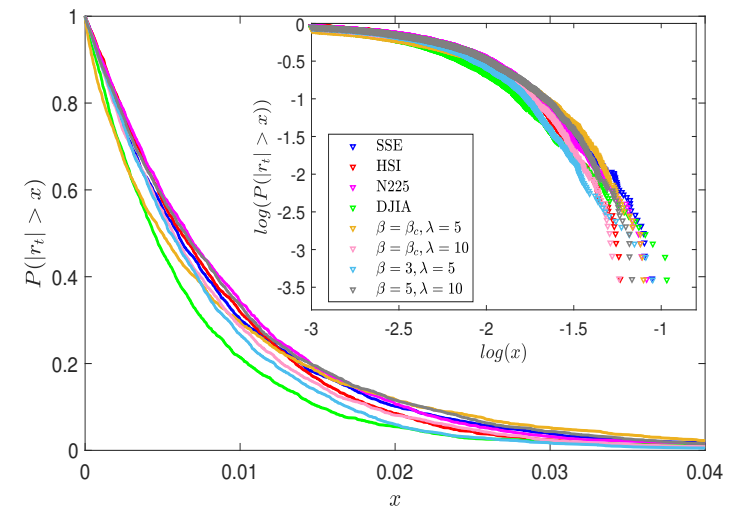

(c)

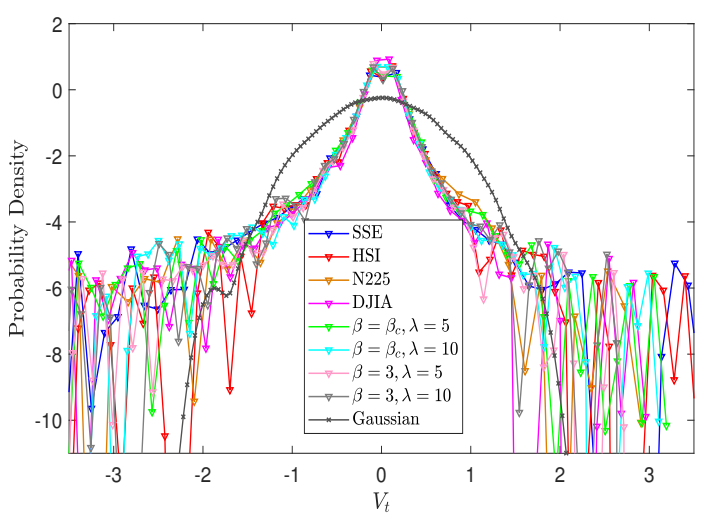

(b)

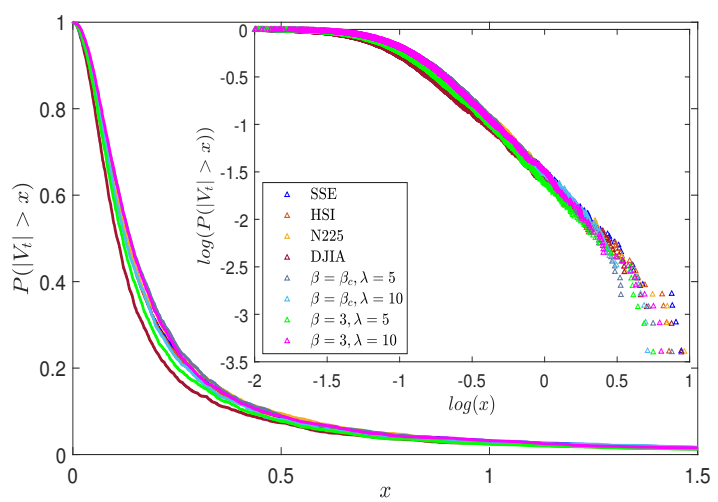

(d)

Figure 4: (a)(b) Logarithmic plots of probability density functions of $r_{t}$ and $V_{t}$. (c)(d) Plots and log-log plots of cumulative distributions for $\left|r_{t}\right|$ and $\left|V_{t}\right|$. 


\section{Complexity behaviors for Potts financial dynamics}

\subsection{Permutation Lempel-Ziv complexity algorithm}

The Lempel-Ziv complexity (LZC) proposed by Lempel and Ziv [33] is a non-parametric estimation of complexity in a one-dimensional time series that is corresponding to the number of distinct substrings and the frequency of their recurrence. The LZC is applied to measure the randomness and complexity of a finite length sequences and is relevant with such theoretical characteristics as entropy and compression ratio. Due to its advantage of capacity of anti-interference to noise, non-parametric needing and easily computing, LZC is applied to measure complexity of dynamical systems in many fields [29, 30, 31, 46]. In this research, we employ a novel method, permutation Lempel-Ziv complexity (PLZC) to study the complexity for the real data and the simulative data from the proposed financial model. For calculating the PLZC, the original series must be transformed into a finite symbol string $s(t)$, this measurement is called coarse-graining. Here we introduce the permutation pattern method to generate symbolic string[31, 46, 47]. For a time series $x(t)(t=1,2, \ldots, N)$, given an embedding dimension $m$ and a time delay $\kappa, x(t)$ is embed to an $m$-dimensional space $X(t)=[x(t), x(t+\kappa), \ldots, x(t+(m-1) \kappa)](t \in$ $1,2, \ldots, N-(m+1) \kappa)$. Then, arrange the components of $X(t)$ in an increasing order $x^{s}\left(t+\left(h_{1}-1\right) \kappa\right) \leq x^{s}\left(t+\left(h_{2}-1\right) \kappa\right) \leq \cdots \leq x^{s}\left(t+\left(h_{m}-1\right) \kappa\right)$. In order to ensure all data points are mapped into one of the possible permutations, when values are equal we order by the index of the data. For example, if $x\left(t+\left(h_{i}-1\right) \kappa\right)=x\left(t+\left(h_{j}-1\right) \kappa\right.$ and $h_{i} \leq h_{j}$, let $x\left(t+\left(h_{i}-1\right) \kappa\right) \leq x\left(t+\left(h_{j}-1\right) \kappa\right.$. Any vector $X(t)$ has a permutation $\pi_{t}=\left[h_{1}, h_{2}, \ldots, h_{m}\right]$,

which is one of permutations of $m$ distinct symbol set $\{1,2, \ldots, m\}$. For each permutation 
$\pi_{l}(l \in\{1,2, \ldots, m !\})$ of symbol set $\{1,2, \ldots, m\}$, it has only one corresponding character $c_{l}\left(c_{l} \in\left\{c_{1}, c_{2}, \ldots, c_{m !}\right\}\right)$. So, the original series $x(t)$ is transformed to a character sequence $s(t)$ with no more than $m$ ! sorts of different characters in. Moreover, $s(t)$ labels the local shape of $x(t)$, since every character in $s(t)$ mirrors a local permutation pattern of $x(t)$.

For a time series $x(t)(t \in 1,2, \cdots, n)$, the PLZC of $x(t)$ are estimated by the following steps $[18,29,30]$ :

(1) Let $S$ and $Q$ represent two subsequences of the original subsequence and $S Q$ be a concatenation of $S$ and $Q$. Here $S Q \pi$ denotes the sequence extracted from $S Q$ in which the last character is deleted.

(2) Let $\varpi(S Q \pi)$ represent the set comprising all the different subsequences of $S Q \pi$.

(3) Set dimension indicator $m$ and time delay parameter $\kappa$. Convert the original series $x(t)$ to a character sequence $s(t)$ whose length is $n$ in accordance with the permutation pattern method.

(4) At the beginning, set the complexity counter $c(n)=1, S=s(1), Q=s(2), S Q=$ $\{s(1), s(2)\}$, and $S Q \pi=s(1)$.

(5) Generally, consider that $S=\{s(1), \cdots, s(r)\}, Q=s(r+1)$, so $S Q \pi=\{s(1), \cdots, s(r)\}$. If $Q \in \varpi(S Q \pi)$, then $Q$ is a subsequence of $S Q \pi$, not a new sequence.

(6) $S$ does not change and renew $Q$ by adding $s(r+2)$ to $Q$, i.e., $Q=\{s(r+1), s(r+2)\}$, then judge whether $Q$ belongs to $\varpi(S Q \pi)$ or not.

(7) Repeat steps (5) and (6) until $Q$ no longer is a part of $\varpi(S Q \pi)$ and $Q=\{s(r+$ $1), \cdots, s(r+i)\}$ is no longer a subsequence of $S Q \pi=\{s(1), \cdots, s(r+i-1)\}$ but a new sequence. We thus add $c(n)$ by one. 
(8) Subsequently $S$ and $Q$ are aggregated and renewed, denoting as $\{s(1), \cdots, s(r+i)\}$, and $s(r+i+1)$.

(9) Repeat the above steps until $Q$ contains the last character. $c(n)$ is the complexity of symbol sequence $s(t)$ which implies the number of distinct new patterns in the original time series.

The total number of subsequences present in $s(t)$ has an upper bound, denoted as $L(n)$

$$
L(n)=c(n)\left[\log _{m !} c(n)+1\right]
$$

Then, the PLZC output is the normalized $c(n)$, defined as

$$
C(n)=\frac{c(n)\left[\log _{m !} c(n)+1\right]}{n}
$$

\subsection{Empirical research by $P L Z C$}

The PLZC complexity behaviors of different volatility sequences for the real data and the simulative data are investigated in this part. In general, many stock traders usually refer to moving averages (MA) prices to make decisions. Then we utilize the MA prices to study the fluctuations of the financial series. The MA prices is defined as follows

$$
r_{t}^{d}=\frac{1}{d} \sum_{k=t}^{t+d-1} r_{k}, \quad t=1,2, \ldots, N-d+1,
$$

where $r_{t}(t \in\{1,2, \ldots, N\})$ is the return series. In addition, the PLZC of the volatility sequences with different exponents, expressed by $\left(r_{t}\right)^{q},\left|r_{t}\right|^{q},\left(V_{t}\right)^{q}$. For different values of $q,(q=1,2, \ldots, 7)$, different series manifest different levels of volatility. We explore the

PLZC values of $r_{t}^{d, q},\left|r_{t}\right|^{d, q}$ and the corresponding sequences $V_{t}^{d, q}$ for the real data and 
Table 2: PLZC value of $r_{t},\left|r_{t}\right|$ and $V_{t}$ for simulative data and real data.

\begin{tabular}{lllllllll}
\hline & SSE & HSI & N225 & DJIA & Simu.1 & Simu.2 & Simu.3 & Simu.4 \\
\hline$r_{t}$ & 0.6394 & 0.6350 & 0.6429 & 0.6290 & 0.6360 & 0.6429 & 0.6307 & 0.6429 \\
$\left|r_{t}\right|$ & 0.6394 & 0.6325 & 0.6464 & 0.9426 & 0.6412 & 0.6360 & 0.6377 & 0.6255 \\
$V_{t}$ & 0.5804 & 0.5769 & 0.5769 & 0.5699 & 0.5682 & 0.5682 & 0.5769 & 0.5682 \\
\hline
\end{tabular}

the simulative data. The parameters for calculating PLZC are set to $m=4$ and $\kappa=1$ respectively.

Figure 5 shows the PLZC analysis results of $r_{t}^{d, q},\left|r_{t}\right|^{d, q}$ and $V_{t}^{d, q}$. Increasing $d$ decreases the PLZC values, indicating that volatility series becomes regular and periodic with the increase of $d$, and that the generation rate of new volatility behaviors also decreases when $d$ increases. For the return series and sequences $V_{t}$, when $q=1$ the PLZC values are much larger than other cases, especially after $d$ is greater than 10. And PLZC did not show obvious fluctuation differences with different $q$ for the absolute returns series $\left|r_{t}\right|$. Furthermore, the PLZC curves of the simulative data are very close to the curves of the real data, indicating the simulative data have the similar complexity behaviors with the real data for fluctuation and volatility dynamics.

Table 2 provides the PLZC values of $r_{t},\left|r_{t}\right|$ and $V_{t}$ of the real data (SSE, HSI, N225 and DJIA) and four groups of simulative data, where 'Simu. 1', 'Simu. 2', 'Simu. 3' and 'Simu. 4' respectively represent the simulative data with $\left\{\beta=\beta_{c}, \lambda=5\right\},\left\{\beta=\beta_{c}, \lambda=10\right\}$, $\{\beta=3, \lambda=5\}$ and $\{\beta=3, \lambda=10\}$. The PLZC of $r_{t}$ for the real data and the simulative data are all around 0.6300 , which means the simulative data exists similar randomness and complexity. The PLZC of $\left|r_{t}\right|$ for DJIA is 0.9426 , other 7 data sets (SSE, HSI, N225, 


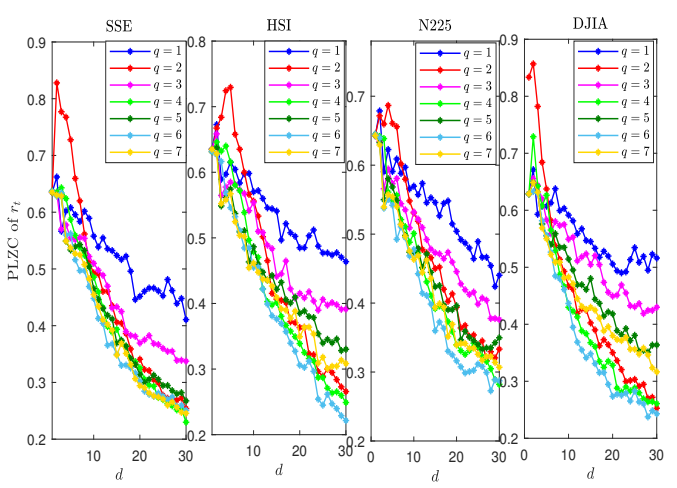

(a)

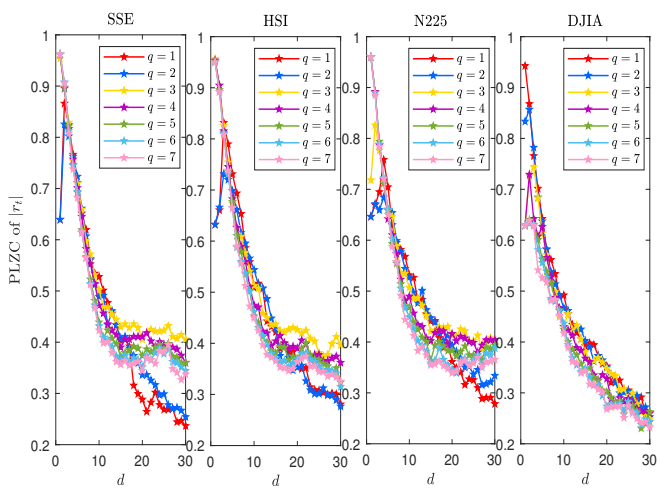

(c)

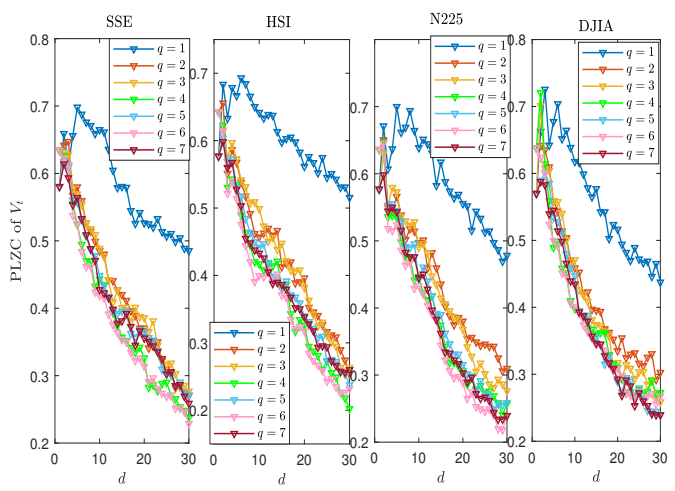

(e)

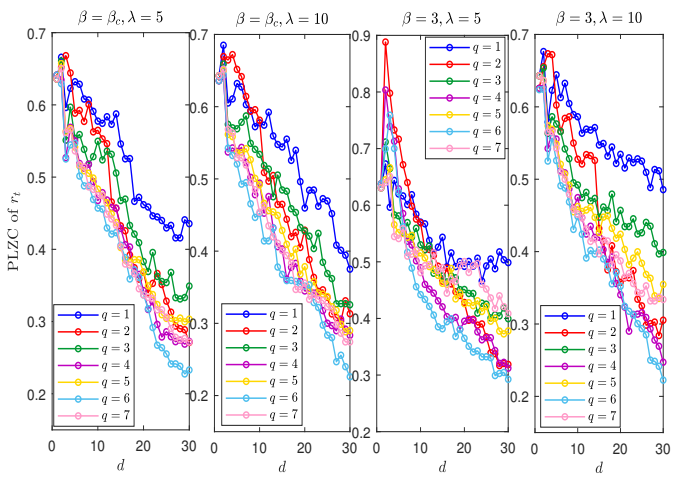

(b)

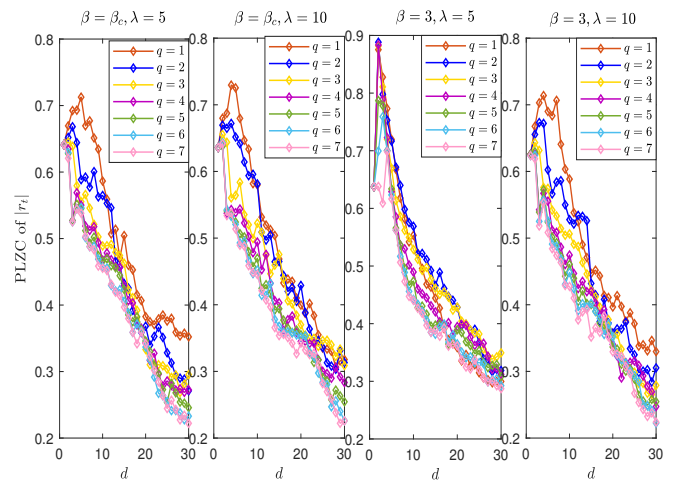

(d)

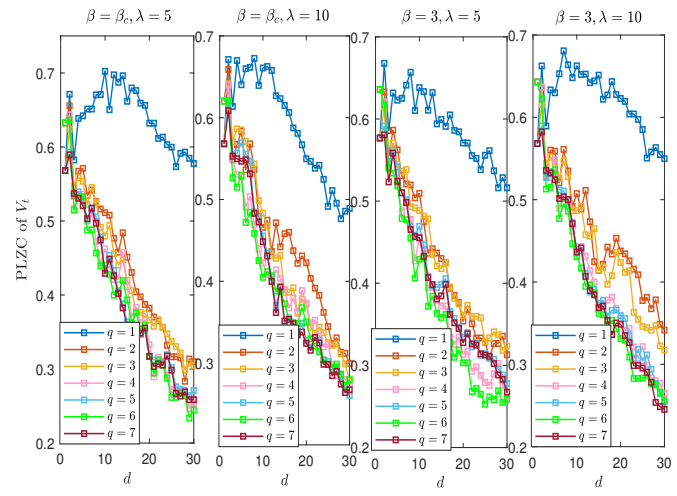

(f)

Figure 5: (a)(b) PLZC plots of $r_{t}^{d, q}$ for real data and simulative data, respectively. (c)(d) PLZC plots of $\left|r_{t}\right|^{d, q}$ for real data and simulative data, respectively. (e)(f) PLZC plots of $V_{t}^{d, q}$ for real data and simulative data, respectively. 
'Simu. 1', 'Simu. 2', 'Simu. 3' and 'Simu. 4' ) are fall in the (0.6250,0.6470). The PLZC of $V_{t}$ for all the data sets are close to 0.5700 . Table 3 reveals the PLZC numerical results of volatility sequences $r_{t},\left|r_{t}\right|$ and $V_{t}$ for moving average. Similarly, 'Simu. 1', 'Simu. 2', 'Simu. 3', 'Simu. 4' in Table 3 stand for the simulative data with $\left\{\beta=\beta_{c}, \lambda=5\right\}$, $\left\{\beta=\beta_{c}, \lambda=10\right\},\{\beta=3, \lambda=5\}$ and $\{\beta=3, \lambda=10\}$ respectively. The PLZC of $r_{t},\left|r_{t}\right|$

and $V_{t}$ decrease with the increase of $d$, this indicates that the MA of volatility sequences become more regular and periodic with the increase of $d$, it also exhibits that there are less new pattern change happening in these series. When $d=5$, the PLZC value of $\left|r_{t}\right|$ is greater than the values of $r_{t}, V_{t}$. It suggests that the randomness and complexity in absolute returns are relatively stronger, followed by the sequence $V_{t}$. The above results show that the series $r_{t},\left|r_{t}\right|$ and $V_{t}$ with different exponents $q$ and $d$ of the real data and the simulative data have similar complexity behaviors for fluctuation and volatility dynamics.

\subsection{Complexity entropy analysis}

In this section, we perform FSE and MFSE methods to investigate the complexity of real stock series and simulative series. Moreover, we make a comparison between the results and discuss their differences and similarities between these different data sets.

\subsubsection{Fractional sample entropy}

Sample entropy, is a method for measure the complexity of dynamics[27]. To explore the characteristics of fractional order information for financial dynamics, a new generalized fractional sample entropy based on the methods in [48] are employed. In general, a small fractional sample entropy value represents large regularity of time series. For a time 
Table 3: PLZC value of $r_{t},\left|r_{t}\right|$ and $V_{t}$ for moving average with $q=1$.

\begin{tabular}{lllllllll}
\hline$r_{t}$ & SSE & HSI & N225 & DJIA & Simu.1 & Simu.2 & Simu.3 & Simu.4 \\
\hline$d=5$ & 0.6090 & 0.6150 & 0.5909 & 0.6073 & 0.6421 & 0.6321 & 0.6199 & 0.6442 \\
$d=10$ & 0.5590 & 0.5702 & 0.5696 & 0.5915 & 0.7022 & 0.5775 & 0.5702 & 0.5863 \\
$d=15$ & 0.5215 & 0.5408 & 0.5460 & 0.5452 & 0.6618 & 0.5442 & 0.5434 & 0.5473 \\
$d=20$ & 0.4515 & 0.4849 & 0.5042 & 0.5057 & 0.6321 & 0.4597 & 0.4920 & 0.5344 \\
$d=25$ & 0.4525 & 0.4770 & 0.4892 & 0.5344 & 0.5997 & 0.4695 & 0.4927 & 0.5250 \\
$d=30$ & 0.4106 & 0.4637 & 0.4404 & 0.5162 & 0.5777 & 0.3751 & 0.4987 & 0.4856 \\
\hline \hline$r_{t} \mid$ & SSE & HSI & N225 & DJIA & Simu.1 & Simu.2 & Simu.3 & Simu.4 \\
\hline$d=5$ & 0.7108 & 0.7312 & 0.7044 & 0.6422 & 0.7126 & 0.7254 & 0.7197 & 0.6900 \\
$d=10$ & 0.5278 & 0.5090 & 0.5314 & 0.4920 & 0.5864 & 0.5798 & 0.5533 & 0.5885 \\
$d=15$ & 0.4031 & 0.4224 & 0.4411 & 0.3923 & 0.5045 & 0.4686 & 0.4444 & 0.4771 \\
$d=20$ & 0.2884 & 0.3659 & 0.3612 & 0.3524 & 0.3826 & 0.3995 & 0.3585 & 0.3966 \\
$d=25$ & 0.2686 & 0.3074 & 0.3254 & 0.3052 & 0.3759 & 0.3351 & 0.3148 & 0.3716 \\
$d=30$ & 0.2368 & 0.2808 & 0.2784 & 0.2596 & 0.3521 & 0.3178 & 0.2997 & 0.3297 \\
\hline \hline$V_{t}$ & SSE & HSI & N225 & DJIA & Simu.1 & Simu.2 & Simu.3 & Simu.4 \\
\hline$d=5$ & 0.6982 & 0.6664 & 0.7000 & 0.6773 & 0.5883 & 0.6403 & 0.6229 & 0.6299 \\
$d=10$ & 0.6648 & 0.6417 & 0.6386 & 0.6177 & 0.5848 & 0.6608 & 0.6382 & 0.6626 \\
$d=15$ & 0.5805 & 0.5976 & 0.6172 & 0.5770 & 0.5810 & 0.6150 & 0.5993 & 0.6512 \\
$d=20$ & 0.5264 & 0.5617 & 0.5554 & 0.5165 & 0.5772 & 0.6498 & 0.5708 & 0.6229 \\
$d=25$ & 0.5068 & 0.5453 & 0.5127 & 0.4720 & 0.5733 & 0.4916 & 0.5558 & 0.5852 \\
$d=30$ & 0.4852 & 0.5156 & 0.4783 & 0.4375 & 0.5695 & 0.4892 & 0.5162 & 0.5550 \\
\hline
\end{tabular}


series $x(t)$ and its $r$-dimensional time-delay embedding expressed as $X_{j}^{r, \varsigma}=\{x(j), x(j+$ $1), \ldots, x(j+(r-1) \varsigma)\}(j=1,2, \ldots, N-r \varsigma)$, where $N$ is the length of the time series, $r$ is the embedding dimension and $\varsigma$ is the time-delay parameter. Two vectors $X_{i}^{r, \varsigma}$ and $X_{j}^{r, \varsigma}$ considered as close if their distance $d\left(X_{i}^{r, \varsigma}, X_{j}^{r, \varsigma}\right)=\max \{|x(i+k \varsigma)-x(j+k \varsigma)|: 0 \leq k \leq$ $r-1\}$ is smaller than a specified tolerance level $\hat{\tau}$, where $i \neq j$ to eliminate self-matches. Let $N_{i}^{r}(\hat{\tau})$ represent the number of vectors $X_{j}^{r, \varsigma}$ that are close to the vector $X_{i}^{r, \varsigma}$. Then the probability that any vector $X_{j}^{r, \varsigma}$ is close to the vector $N_{i}^{r}(\hat{\tau})$ within a tolerance level $\hat{\tau}$ is described as

$$
C^{r}(\hat{\tau})=\frac{1}{n-r} \sum_{i}^{n-r} \frac{N_{i}^{r}(\hat{\tau})}{(n-r-1)} .
$$

The sample entropy of time series $x(t)$ is given by

$$
H_{\text {SampEn }}(x, r, \hat{\tau})=-\ln \left[\frac{C^{r+1}(\hat{\tau})}{C^{r}(\hat{\tau})}\right]
$$

Then the fractional sample entropy of time series $x(t)$ is defined as

$$
H_{F S E}(x, r, \alpha, \hat{\tau})=-\frac{\ln C^{r+1}(\hat{\tau})-\ln C^{r}(\hat{\tau})+\psi(1)-\psi(1-\alpha)}{\Gamma(\alpha+1)} \times\left[\frac{C^{r+1}(\hat{\tau})}{C^{r}(\hat{\tau})}\right]^{-\alpha}
$$

$r$ and $\hat{\tau}$ are critical in calculating the value of sample entropy, we here set parameters $r=2$ and $\hat{\tau}=l \times S D(l=0.15)$, where SD is the standard deviation of the original time series $[32,49]$.

\subsubsection{Multiscale fractional sample entropy}

The complexity of dynamic sequences is not limited to the single scale. In this study, 
we improve an approach called multiscale fractional sample entropy (MFSE), which takes into account the multiple time scales based on the fractional sample entropy theory.

Step 1: For one-dimensional time series $\left\{x_{t}\right\}(t \in 1,2, \ldots, N)$, construct the coarsegrained time series $y_{j}^{(\tau)}, \tau$ is the scale factor[50, 51], according to the equation below

$$
y_{j}^{(\tau)}=\frac{1}{\tau} \sum_{i=(j-1) \tau+1}^{j} x_{i}, 1 \leq j \leq\left[\frac{N}{\tau}\right] .
$$

Step 2: The multiscale fractional sample entropy is computed for each coarse-grained time series by using the FSE analysis, then the complexity entropy of MFSE can be researched as the function of scale factor $\tau$.

\subsection{Empirical results by FSE and MFSE}

The FSE and MFSE analysis are applied to measure the complexity behaviors of the financial stock data (SSE, HSI, N225 and DJIA) and the simulative data from the proposed Potts financial model with parameter $\left\{\beta=\beta_{c}, \lambda=5\right\},\left\{\beta=\beta_{c}, \lambda=10\right\},\{\beta=3, \lambda=5\}$ and $\{\beta=3, \lambda=10\}$. Fig. 6 depicts the FSE plots versus parameter $\alpha$ of volatility sequences $r_{t},\left|r_{t}\right|$ and $V_{t}$ for real stock data and simulative data, where $\alpha$ increases from -1 to 0.6 . The corresponding concrete results with $\alpha=-0.8,-0.5,-0.2,0,0.2,0.5$ are showed in Table 4. In Fig. 6, the FSE entropy values for these volatility sequences first ascend with the increase of $\alpha$, and then starts to descend after reaching the maximum value. The return series get the maximum value when $\alpha$ is around 0.4 , the absolute return series takes the maximum value when $\alpha$ is close to 0.3 and the $V_{t}$ sequences achieves the maximum value when $\alpha$ is in the vicinity of 0.2. Moreover, the FSE measures of DJIA for the three volatility sequences exhibit the lowest degree of complexity. The complexity 
and randomness of simulative data with parameter $\beta=\beta_{c}, \lambda=5$ is very similar to DJIA. For the return series and the absolute series, HSI has the highest complexity than other 7 data sets, and as $\alpha$ increases, the complexity intensity decreases relatively slowly. The complexity of simulative data with parameter $\beta=\beta_{c}, \lambda=10, \beta=3, \lambda=5$ and $\beta=3, \lambda=10$ is in the middle of the complexity of SSE and HSI. For the sequences $V_{t}$, the simulative data and the real data shows almost uniform complexity. From Table 4, the FSE reveals more differences for volatility sequences when $\alpha>0$, but when $\alpha<0$ the complexity of the volatility sequences has approximate values. The FSE values for all the real data and the simulative data are in $[0.12,0.19]$ when $\alpha=-0.8$. When $\alpha=0.5$, the FSE values of return series are in $[0.8,2.9]$, and for the sequences $V_{t}$, the FSE values is between $[-0.4,-0.1]$.

Fig. 7 displays the MFSE curves for volatility sequences of the real financial stock data (SSE, HSI, N225 and DJIA) and the simulative data from the proposed Potts financial model with parameter $\left\{\beta=\beta_{c}, \lambda=5\right\},\left\{\beta=\beta_{c}, \lambda=10\right\},\{\beta=3, \lambda=5\}$ and $\{\beta=3, \lambda=10\}$. It can be seen from Fig 7 , the MFSE results of different volatility series vary as the scale factor $\tau$ increases, indicating the multiscale structure of the complexity and the necessity of studying the financial time series on multiple scales. With the scale factor increases, the MFSE complexity fluctuates sharply. For return series $r_{t}$ of simulation data and real data, the complexity fluctuations are more severe than the other two volatility sequences. The complexity behavior of $\left|r_{t}\right|$ changes relatively slightly as the scale factor increase, but fluctuation is also beginning to be obvious after $\tau>20$. The MFSE complexity of sequences $V_{t}$ has the similar results with $\left|r_{t}\right|$, both for the real data 


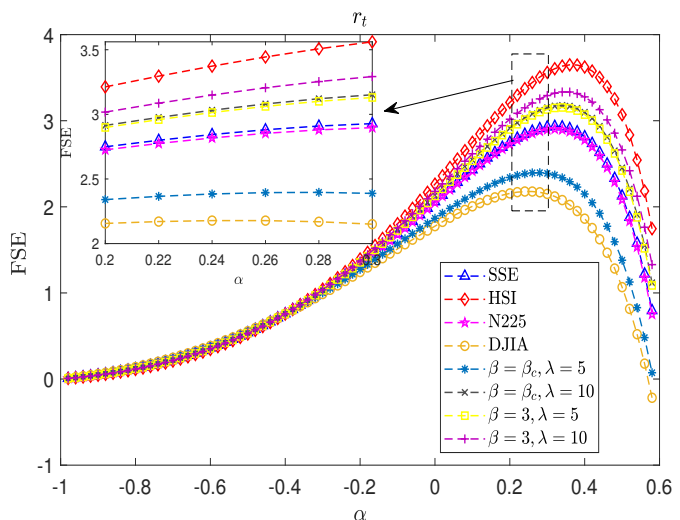

(a)

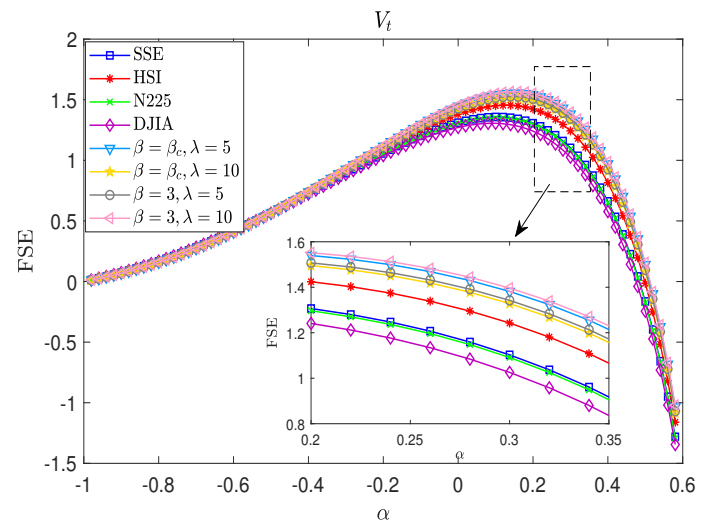

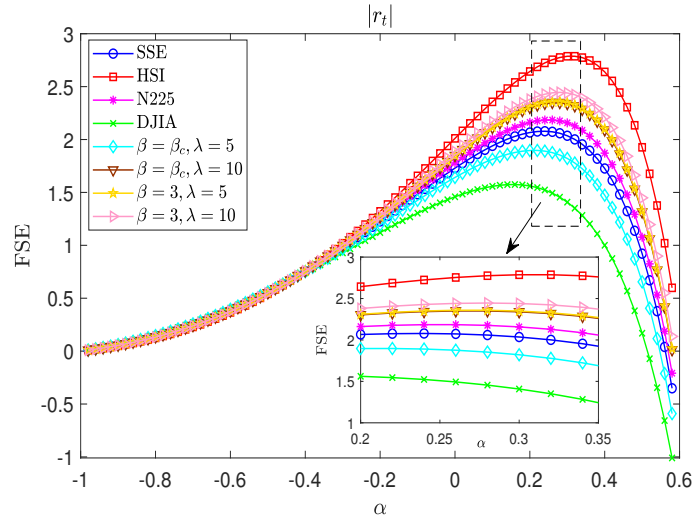

(b)

(c)

Figure 6: (a)(b)(c) FSE curves versus $\alpha$ of volatility sequences $r_{t},\left|r_{t}\right|$ and $V_{t}$ for real stock data and simulative data. 
Table 4: FSE analysis of $r_{t},\left|r_{t}\right|$ and $V_{t}$ for simulative data and real financial data.

\begin{tabular}{lllllll}
\hline$r_{t}$ & $\alpha=-0.8$ & $\alpha=-0.5$ & $\alpha=-0.2$ & $\alpha=0$ & $\alpha=0.2$ & $\alpha=0.5$ \\
\hline SSE & 0.1405 & 0.5790 & 1.4029 & 2.1352 & 2.7995 & 1.8788 \\
HSI & 0.1282 & 0.5654 & 1.4711 & 2.3597 & 3.2955 & 2.8350 \\
N225 & 0.1411 & 0.5796 & 1.3991 & 2.1235 & 2.7747 & 1.8336 \\
DJIA & 0.1582 & 0.5926 & 1.2959 & 1.8255 & 2.1703 & 0.8148 \\
$\beta=\beta_{c}, \lambda=5$ & 0.1524 & 0.5890 & 1.3319 & 1.9294 & 2.3654 & 1.1267 \\
$\beta=\beta_{c}, \lambda=10$ & 0.1360 & 0.5744 & 1.4286 & 2.2169 & 2.9761 & 2.2074 \\
$\beta=3, \lambda=5$ & 0.1364 & 0.5748 & 1.4262 & 2.2093 & 2.9593 & 2.1757 \\
$\beta=3, \lambda=10$ & 0.1332 & 0.5713 & 1.4338 & 2.2669 & 3.0836 & 2.4193 \\
\hline \hline$\left|r_{t}\right|$ & $\alpha=-0.8$ & $\alpha=-0.5$ & $\alpha=-0.2$ & $\alpha=0$ & $\alpha=0.2$ & $\alpha=0.5$ \\
\hline SSE & 0.1611 & 0.5940 & 1.2774 & 1.7762 & 2.0756 & 0.6694 \\
HSI & 0.1435 & 0.5818 & 1.3856 & 2.0819 & 2.6868 & 1.6759 \\
N225 & 0.1580 & 0.5925 & 1.2971 & 1.8288 & 2.1756 & 0.8245 \\
DJIA & 0.1781 & 0.5977 & 1.1586 & 1.4840 & 1.5456 & -0.0719 \\
$\beta=\beta_{c}, \lambda=5$ & 0.1667 & 0.5962 & 1.2403 & 1.6807 & 1.8968 & 0.4054 \\
$\beta=\beta_{c}, \lambda=10$ & 0.1536 & 0.5898 & 1.3248 & 1.9049 & 2.3257 & 1.0618 \\
$\beta=3, \lambda=5$ & 0.1534 & 0.5896 & 1.3262 & 1.9087 & 2.3331 & 1.0740 \\
$\beta=3, \lambda=10$ & 0.1512 & 0.5882 & 1.3394 & 1.9459 & 2.4075 & 1.1961 \\
\hline \hline$V_{t}$ & $\alpha=-0.8$ & $\alpha=-0.5$ & $\alpha=-0.2$ & $\alpha=0$ & $\alpha=0.2$ & $\alpha=0.5$ \\
\hline SSE & 0.1871 & 0.5955 & 1.0876 & 1.3261 & 1.2801 & -0.3962 \\
HSI & 0.1829 & 0.5969 & 1.1215 & 1.4000 & 1.4026 & -0.2505 \\
N225 & 0.1874 & 0.5953 & 1.0847 & 1.3198 & 1.2699 & -0.4080 \\
DJIA & 0.1894 & 0.5943 & 1.0680 & 1.2842 & 1.2120 & -0.4741 \\
$\beta=\beta_{c}, \lambda=5$ & 0.1789 & 0.5976 & 1.1529 & 1.4707 & 1.5229 & -0.1010 \\
$\beta=\beta_{c}, \lambda=10$ & 0.1804 & 0.5974 & 1.1410 & 1.4438 & 1.4767 & -0.1592 \\
$\beta=3, \lambda=5$ & 0.1800 & 0.5975 & 1.1442 & 1.4510 & 1.4890 & -0.1437 \\
$\beta=3, \lambda=10$ & 0.1784 & 0.5977 & 1.1561 & 1.4783 & 1.5358 & -0.0844 \\
\hline
\end{tabular}


and the simulative data. From the empirical results of Fig. 6 and Fig. 7, for the return series, the absolute return series and the sequences $V_{t}$, the FSE and MFSE complexity entropy of the simulative data are approximate to those of real financial data.

Table 5 provide the numerical results of the MFSE analysis, where $\tau=1,5,10$. The table shows that the MFSE values of return series $r_{t}$ are much larger than the values of $\left|r_{t}\right|$ and $V_{t}$ of the same scale factor $\tau$. In the case of $\tau=1$, the MFSE values of $r_{t},\left|r_{t}\right|$ and $V_{t}$ for the simulative data from the proposed financial model are very close to the real financial data. When $\tau=5$, the MFSE numerical results of return series for the real data and the simulative data nearly fluctuate in $[2.2162,2.8590]$. For the sequences $V_{t}$, the MFSE values of simulative data is a little bigger that the real data. When $\tau=10$, the complexity of $\left|r_{t}\right|$ for real data SSE, DJIA and the simulative data $\beta=3, \lambda=5$ are smaller than the other five data sets, which means that the MFSE analysis for the simulative data and the real data are strongly persistent. In summary, the return series shows higher complexity and randomness for both the simulative data and real data, the complexity of $V_{t}$ at $\tau=1$ has no obvious difference for the simulative data and real data. The simulative data from the proposed stochastic Potts model has the similar MFSE complexity behaviors for different scale factor $\tau$ with the real stock data.

\section{Conclusion}

In this study, a new stochastic financial price model is developed based on Potss dynamics and compound Poisson process. The new model considers the fluctuations formation mechanism by combining the information interaction among traders with the 


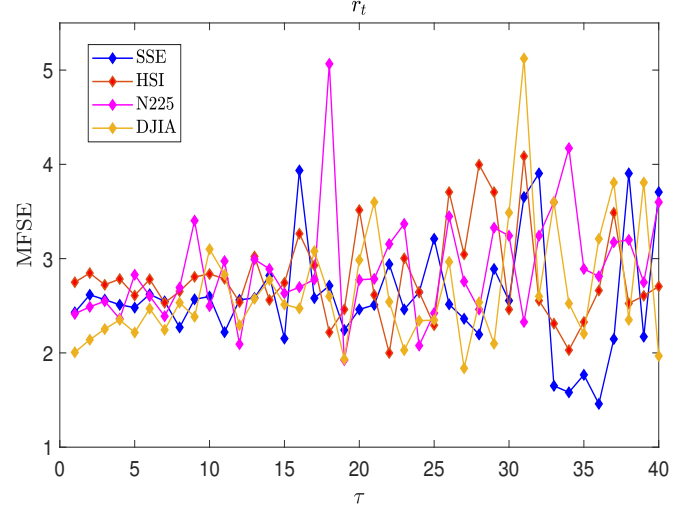

(a)

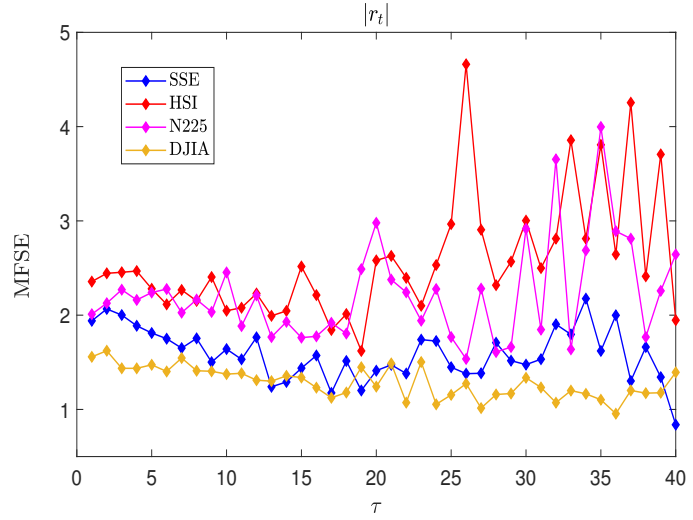

(c)

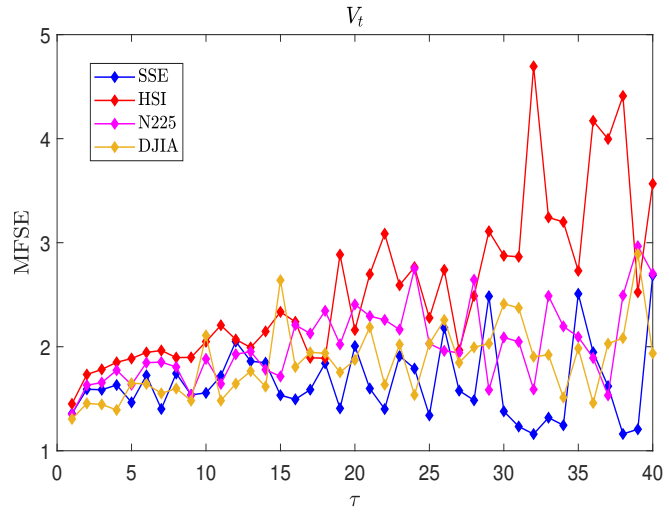

(e)

Figure 7: (a)(b) MFSE plots of $r_{t}$ for real data and simulative data. (c)(d) MFSE plots of $\left|r_{t}\right|$ for real data and simulative data. (e)(f) MFSE plots of $V_{t}$ for real data and simulative data.

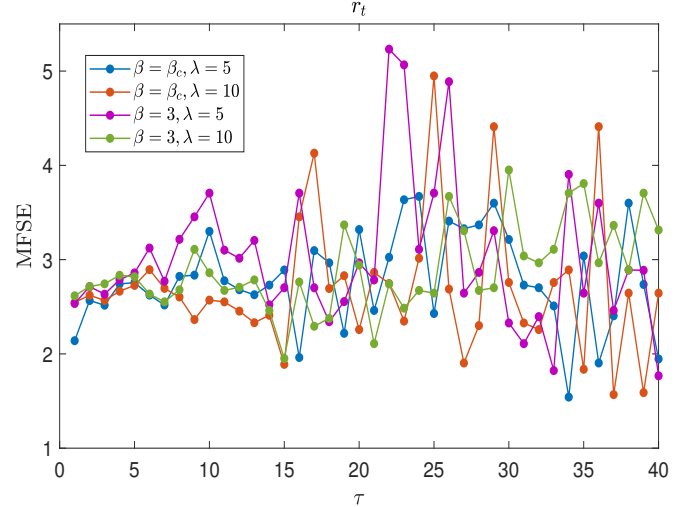

(b)

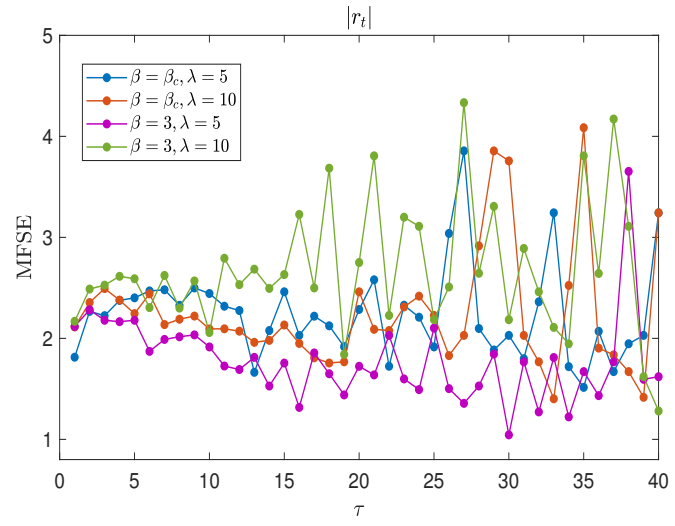

(d)

(f)

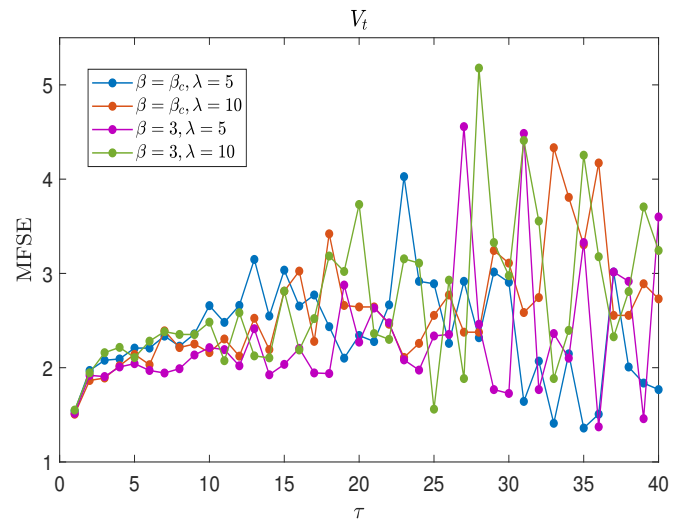


Table 5: MFSE value of $r_{t},\left|r_{t}\right|$ and $V_{t}$ for simulative data and real financial data.

\begin{tabular}{|c|c|c|c|c|c|c|c|c|c|}
\hline \multirow[b]{2}{*}{ Data } & \multicolumn{3}{|c|}{$\tau=1$} & \multicolumn{3}{|c|}{$\tau=5$} & \multicolumn{3}{|c|}{$\tau=10$} \\
\hline & $r_{t}$ & $\left|r_{t}\right|$ & $\overline{V_{t}}$ & $r_{t}$ & $\left|r_{t}\right|$ & $\overline{V_{t}}$ & $r_{t}$ & $\left|r_{t}\right|$ & $V_{t}$ \\
\hline SSE & 2.4305 & 1.9399 & 1.3578 & 2.4801 & 1.8107 & 1.4648 & 2.5978 & 1.6402 & 1.5563 \\
\hline HSI & 2.7494 & 2.3562 & 1.4509 & 2.6088 & 2.2798 & 1.8867 & 2.8364 & 2.0470 & 2.0483 \\
\hline $\mathrm{N} 225$ & 2.4142 & 2.0102 & 1.3500 & 2.8290 & 2.2400 & 1.6352 & 2.4962 & 2.4535 & 1.8831 \\
\hline DJIA & 2.0059 & 1.5578 & 1.3054 & 2.2162 & 1.4735 & 1.6492 & 3.1001 & 1.3749 & 2.1089 \\
\hline$\beta=\beta_{c}, \lambda=5$ & 2.1402 & 1.8133 & 1.5409 & 2.7559 & 2.4012 & 2.2080 & 3.2998 & 2.4442 & 2.6584 \\
\hline$\beta=\beta_{c}, \lambda=10$ & 2.5455 & 2.1130 & 1.5065 & 2.7266 & 2.2448 & 2.1415 & 2.5707 & 2.0933 & 2.1602 \\
\hline$\beta=3, \lambda=5$ & 2.5347 & 2.1182 & 1.5157 & 2.8590 & 2.1791 & 2.0428 & 3.7055 & 1.9151 & 2.2127 \\
\hline$\beta=3, \lambda=10$ & 2.6165 & 2.1688 & 1.5506 & 2.8186 & 2.5902 & 2.1073 & 2.8606 & 2.0579 & 2.4834 \\
\hline
\end{tabular}

randomness coming from the financial system and the uncertain facts. Then we employ a novel statistics volatility duration average intensity sequences $V_{t}$ to explore the fluctuation and volatility behaviors of the simulative data from the proposed model and the real stock data. Meanwhile, the classical volatility statistics including return series $r_{t}$ and absolute return series $\left|r_{t}\right|$ are also investigated to research on the price fluctuation and volatility dynamics of the proposed model. Compared with the real stock data of SSE, HSI, N225 and DJIA, the simulative data with parameter $\left\{\beta=\beta_{c}, \lambda=5\right\},\left\{\beta=\beta_{c}, \lambda=10\right\}$, $\{\beta=3, \lambda=5\}$ and $\{\beta=3, \lambda=10\}$ derived from the proposed model are selected for the analysis of statistical properties and complexity behaviors. The empirical results of Descriptive statistics, Kolmogorov-Smirnov test, probability density function and cumulative distribution function indicate that the volatility sequences $r_{t},\left|r_{t}\right|$ and $V_{t}$ of all simulative data do not follow the Gaussian distribution, displaying significant features of excess kurtosis and fat tail, which are conforming to the relevant features of the real data. Besides, it can be obtained the sequences $r_{t}$ and $V_{t}$ obey the power-law distribution 
$P\left(\left|r_{t}\right|>x\right) \sim x^{-\nu}$, and for the return series $\nu \approx 3$ for sequences $V_{t} \nu \approx 1.6$. Furthermore, the complexity behaviors of different volatility are discussed by applying PLZC, FSE and MFSE analysis. The PLZC results show that the volatility sequences $r_{t},\left|r_{t}\right|$ and $V_{t}$ with different parameter $q$ and $d$ of the real data and the simulative data have comparable permutation complexity for volatility dynamics. Especially for $r_{t}$ and $V_{t}$, the PLZC values with $q=1$ are much larger than other exponent cases. The FSE reveals more differences for volatility sequences when $\alpha>0$, for $\alpha<0$, the complexity of the volatility sequences has approximate values. The MFSE results of different volatility series vary as the scale factor $\tau$ increases for all the real data and simulative data, which implies the indispensability of studying the financial time series on multiple scales. The empirical conclusions of the above statistical attributes and complexity analysis proves that the financial price model is feasible, and it can beneficially reconstruct the volatility dynamics of real stock markets to some extent.

Acknowledgements: This research was supported by North China University of Technology (Grant No.110051360002).

\section{Compliance with ethical standards}

Conflicts of Interest: The authors declare no conflict of interest.

\section{References}

[1] Farmer, J.D. ; Joshi, S. The price dynamics of common trading strategies. J. Econ. Behav. Organ. 2002, 49 : 149-171. 
[2] Lux, T. ; Marchesi, M. Scaling and criticality in a stochastic multiagent model of a financial market. Nature. 1999, 397 : 498-500.

[3] Bouchaud, J.P. ; Potters, M. Theory of Financial risk and derivative pricing: from statistical physics to risk management. Cambridge University Press, Cambridge, 2003.

[4] Stanley,H.E.; Gabaix, X. ; Gopikrishnan, P. ; Plerou, V. Economic fluctuations and statistical physics: the puzzle of large fluctuations. Nonlinear Dynam. 2006, 44: $329-340$.

[5] Gontis, V.; Havlina, S.; Kononovicius, A. ; et al. Stochastic model of financial markets reproducing scaling and memory in volatility return intervals. Phyisca A. 2016, 462: 1091-1102.

[6] Gabaix, X.; Gopikrishanan, P. ;Plerou, V.; Stanley, H.E. A theory of power-law distributions in financial market fluctuations. Nature. 2003, 423 : 267-270.

[7] Calvet, L.; Fisher, A. Multifractal Volatility: Theory, Forecasting, and Pricing. Academic Press: New York, USA, 2008.

[8] Grech, D.; Pamula, G. Multifractality of nonlinear transformations with application in finances. Acta Phys. Pol. A. 2013, 123 : 529-537.

[9] Amaral, L.A.N. ; Buldyrev, S.V.; Havlin, S.; et al. Power law scaling for a system of interacting units with complex internal structure. Phys. Rev. Lett. 1998, 80 : $1385-1388$. 
[10] Wang, J.; Wang, J. Cross-correlation complexity and synchronization of the financial time series on Potts dynamics. Physica A. 2020, 541: 123286.

[11] Li, J.; Shang, P. ; Zhang, X. Financial time series analysis based on fractional and multiscale permutation entropy. Communications in Nonlinear Science and Numerical Simulation. 2019, 78: 104880.

[12] Lux, T. Estimation of an agent-based model of investor sentiment formation in financial markets. J. Econ. Dyn. Control. 2012, 36 :1284-1302.

[13] Boswijk,H. P. ; Hommes, C. H.; Manzan,S. Behavioral heterogeneity in stock prices. Journal of Economic dynamics and control. 2007, 31(6): 1938-1970.

[14] Durrett, R. Lecture Notes on Particle Systems and Percolation. Wadsworth \& Brooks, Pacific Grove-California, 1998.

[15] Zivot, E. ; Wang, J.H. Modeling Financial Time Series with SPLUS. Springer, New York, 2006.

[16] Mike, S. ; Farmer, J.D. An empirical behavioral model of liquidity and volatility. J. Econ. Dyn. Control. 2008, 32 : 200-234.

[17] Stauffer, D. ; Penna, T.J.P. Crossover in the Cont-Bouchaud percolation model for market fluctuations. Physica A. 1998, 256 : 284-290.

[18] Wang, J. ; Wang, J. ; Stanley, H. E. Multiscale multifractal DCCA and complexity behaviors of return intervals for Potts price model. Physica A. 2018, 492: 889-902. 
[19] Wang,Y.; Zheng, S.; Zhang, W. ; et al. Modeling and complexity of stochastic interacting Lévy type financial price dynamics. Physica A. 2018, 499: 498-511.

[20] Zhang, B. ; Wang, G. ;Wang, Y. ; et al. Multiscale statistical behaviors for Ising financial dynamics with continuum percolation jump. Physica A. 2019, 525: 10121025.

[21] Wang, J. ; Wang, J. Measuring the correlation complexity between return series by multiscale complex analysis on Potts dynamics. Nonlinear Dynamics. 2017, 89 : 2703- 2721.

[22] Bornholdt, S. ; Wagner, F. Stability of money: phase transitions in an Ising economy. Physica A. 2002, 316: 453-468.

[23] Wang, F. ; Yamasaki, K. ; Havlin, S. ; Stanley, H.E. Scaling and memory of intraday volatility return intervals in stock markets. Phys. Rev. E. 2006, 73: 026117.

[24] Yang, G.; Wang, J.; Deng, W. Nonlinear analysis of volatility duration financial series model by stochastic interacting dynamic system. Nonlinear Dynamics. 2015, 80(1): 701-713.

[25] Wang, H.; Wang, J. ; Wang, G. Nonlinear continuous fluctuation intensity financial dynamics and complexity behavior. Chaos: An Interdisciplinary Journal of Nonlinear Science. 2018, 28(8): 083122.

[26] Li,R.; Wang, J. Symbolic complexity of volatility duration and volatility difference component on voter financial dynamics. Digital Signal Processing. 2017, 63: 56-71. 
[27] Costa, M. ;Goldberger, A. L. ; Peng, C. K. Multiscale entropy analysis of biological signals. Physical review E. 2005, 71(2): 021906.

[28] Wu, Y. ; Shang, P.; Li, Y. Multiscale sample entropy and crosssample entropy based on symbolic representation and similarity of stock markets. Communications in Nonlinear Science and Numerical Simulation. 2018, 56: 49-61.

[29] Hong, H. ; Liang, M. Fault severity assessment for rolling element bearings using the Lempel-Ziv complexity and continuous wavelet transform. J. Sound Vib. 2009, 320: $452-468$.

[30] Fernández, A.; LópezIbor, M.I. ; Turrero, A. ; et al. Lempel-Ziv complexity in schizophrenia: a MEG study. Clinical neurophysiology. 2011,122: 2227-2235.

[31] Bai, Y. ; Liang, Z.; Li, X. A permutation Lempel-Ziv complexity measure for EEG analysis. Biomedical Signal Processing and Control. 2015, 19: 102-114.

[32] Xu, K.; Wang, J. Weighted fractional permutation entropy and fractional sample entropy for nonlinear Potts financial dynamics. Physics Letters A, 2017, 381(8): 767-779.

[33] Lempel, A. ; Ziv, J. On the complexity of finite sequences, Inform. Theory IEEETrans. 1976, 22: 75-81.

[34] Gliozzi, F. Simulation of potts models with real $q$ and no critical slowing down. Phys. Rev. E. 2002, 66: 016115. 
[35] Grollau, S. ; Rosinberg, M.L.; Tarjus, G. The ferromagnetic q- state Potts model on three-dimensional lattices: a study for real values of $q$. Physica A. 2001, 296: 460-482.

[36] Deng, Y. ; Blóte, H.W.J. ; Nienhuis, B. Backbone exponents of the two-dimensional q-state Potts model: a Monte Carlo investigation. Phys. Rev. E. 2004, 69: 026114.

[37] Cont, R.; Tankov, P. Financial modeling with jump processes, Chapman \& HallI CRC: USA, 2004.

[38] Tosun, P. D.; Abásolo, D. ; Stenson, G. ; et al. Characterisation of the Effects of Sleep Deprivation on the Electroencephalogram Using Permutation Lempel-Ziv Complexity, a Non-Linear Analysis Tool. Entropy, 2017, 19(12): 673.

[39] Tsay, R. S. Analysis of Financial Time Series. John Wiley \& Sons, Hoboken, USA, 2005.

[40] Ross, S.M. An Introduction to Mathematical Finance. Cambridge University Press, Cambridge, 1999.

[41] Mantegna, R.N. ; Stanley, H.E. An Introduction to Econophysics: Correlations and Complexity in Finance. Cambridge University Press, Cambridge, 1999.

[42] Tangmongkollert, K. ; Suwanna, S. Modeling of price and profit in coupled-ring networks. Eur. Phys. J. B. 2016, 89:146. 
[43] Lux, T.; Marchesi, M. Scaling and criticality in a stochastic multiagent model of a financial market. Nature. 1999,397: 498-500.

[44] Podobnik, B. ; Fu,D.F. ; Stanley,H.E. ; Ivanov, P.C. Power-law auto-correlated stochastic processes with long-range crosscorrelations. Eur. Phys. J. B. 2007, 56: $47-52$.

[45] Weber, M.D. ; Leemis, L. M. ; Kincaid, R. K. Minimum Kolmogorov- Smirnov test statistic parameter estimates. Journal of Statistical Computation and Simulation. 2006, 76(3): 195-206.

[46] Li, R. ; Wang, J. Interacting price model and fluctuation behavior analysis from Lempel-Ziv complexity and multi-scale weightedpermutation entropy. Phys. Lett. A. 2016: 380: 117-129.

[47] Bandt, C.; Pompe, B. Permutation entropy: a natural complexity measure for time series, Phys. Rev. Lett. 2002, 88 : 174102.

[48] Machado. J.T. Fractional order generalized information. Entropy, 2014, 16(4): 23502361.

[49] Zunino, L. ; Pérez, D.G. ; Martín, M.T. ; Garavaglia, M.; Plastino, A.; Rosso, O.A. Permutation entropy of fractional Brownian motion and fractional Gaussian noise, Phys. Lett. A. 2008, 372: 4768-4774.

[50] Costa, M. ; Goldberger, A.L. ; Peng, C.K. Multiscale entropy analysis of complex physiological time series, Phys. Rev. Lett. 2002, 89 : 068102. 
[51] Thuraisingham, R.A. ; Gottwald, G.A. On multiscale entropy analysis for physiological data, Physica A. 2006, 366: 323-332. 


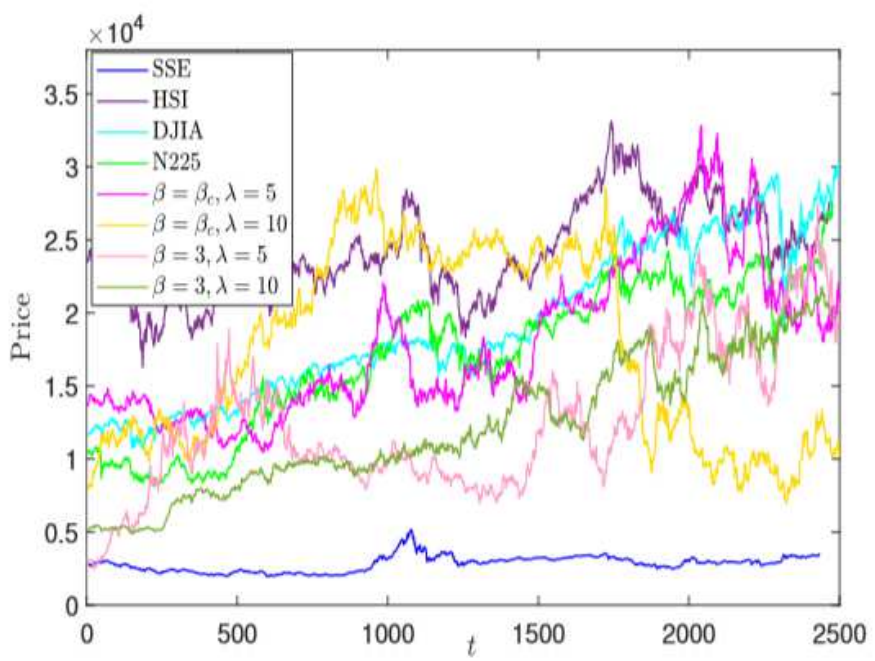

(a)

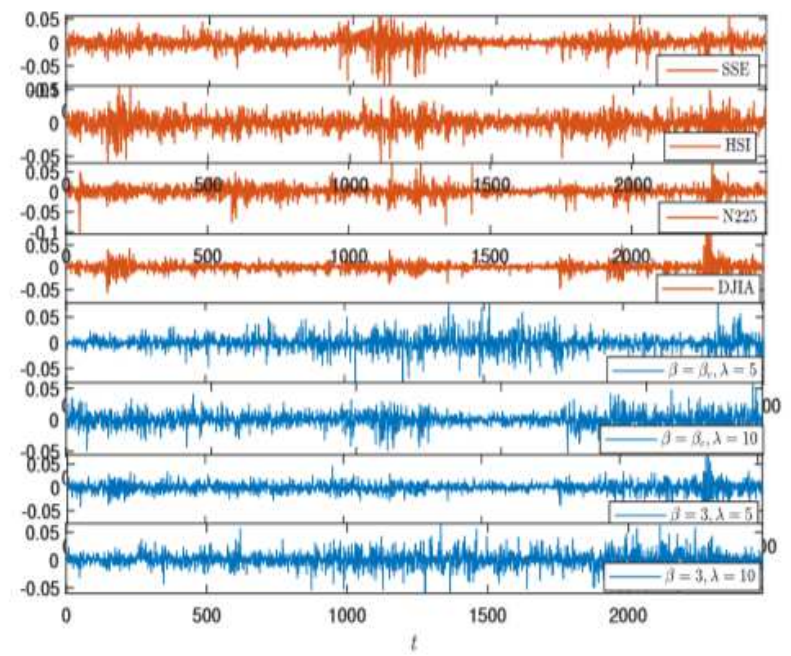

(b)

\section{Figure 1}

(a) Price time series of real indexes and the simulative data of the stochastic Potts financial model. (b) Plots of return series it for real indexes and the simulative data of the stochastic Potts financial model.

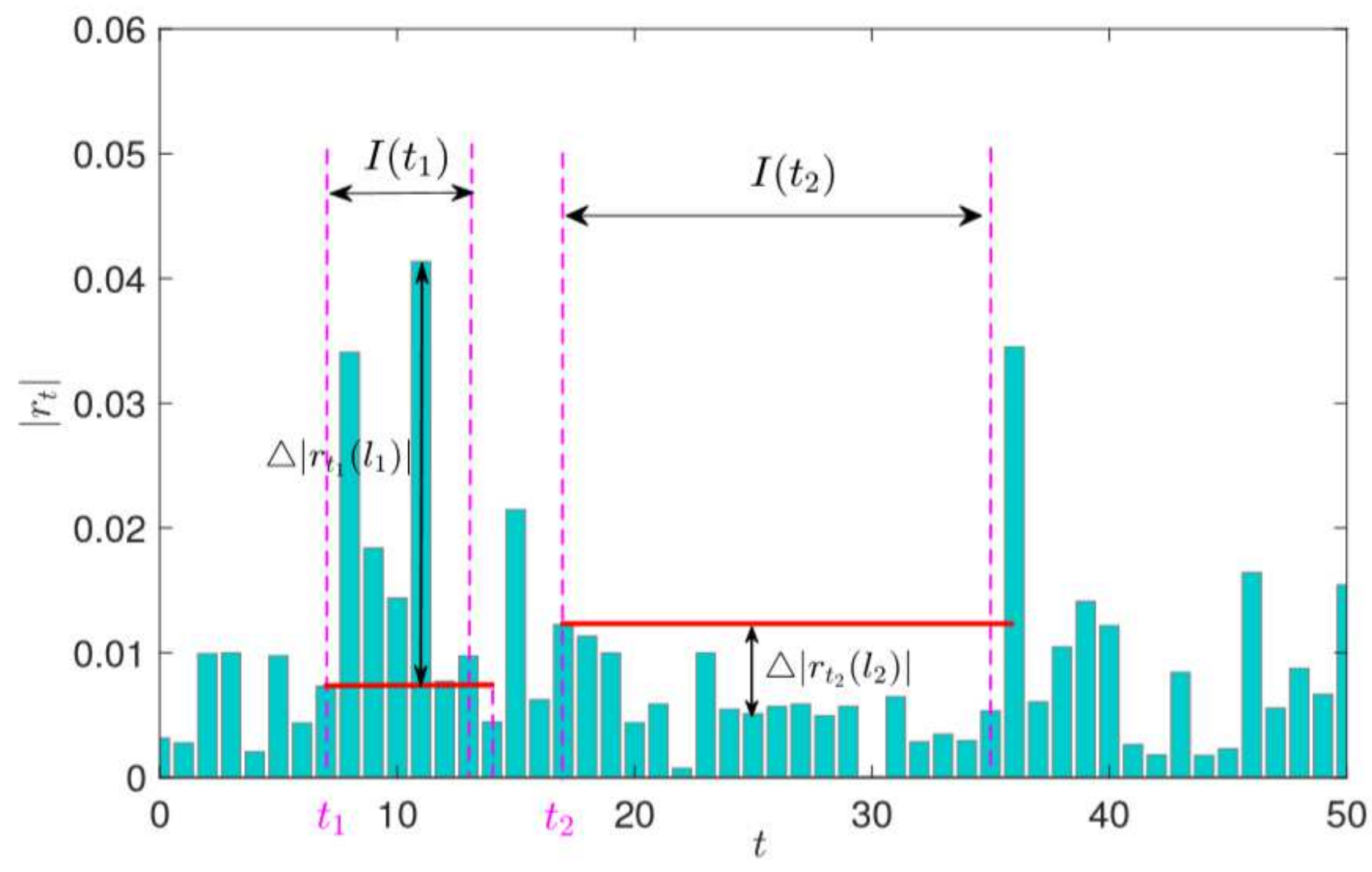


Figure 2

Illustrations of I(t), $\Delta|\mathrm{rt}(\mathrm{i})|$ in the volatility series.

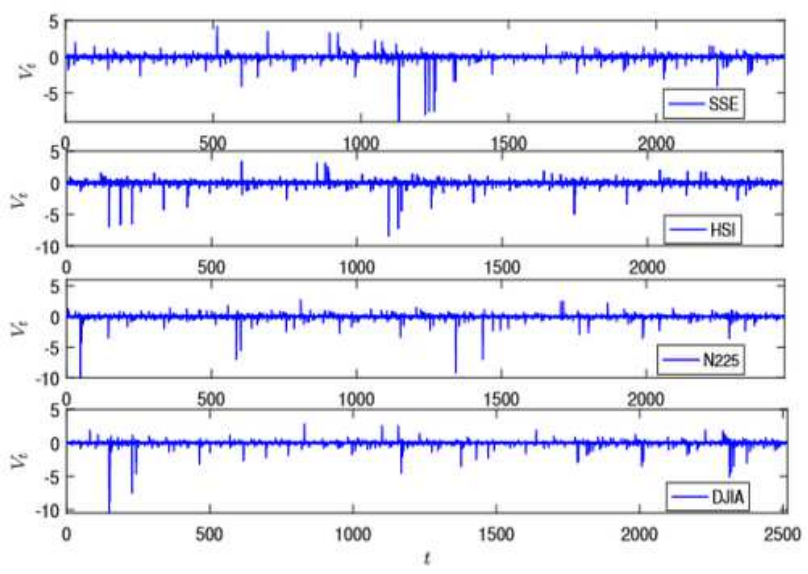

(a)

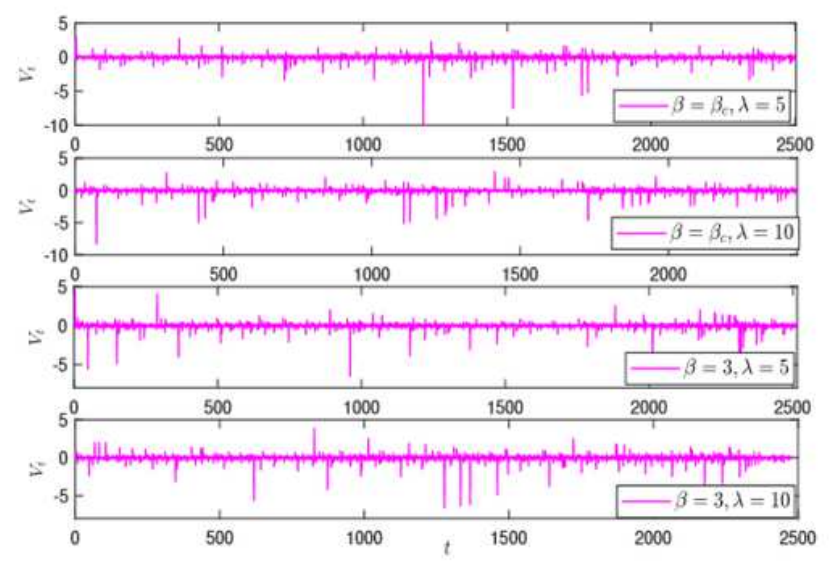

(b)

\section{Figure 3}

(a) The volatility duration average intensity sequences Vt of real data for different market indexes. (b) The volatility duration average intensity sequences $V t$ sequences of the simulative data with $\{\beta=\beta c, \lambda=5\},\{\beta$ $=\beta c, \lambda=10\},\{\beta=3, \lambda=5\},\{\beta=3, \lambda=10\}$ for the stochastic Potts financial model. 


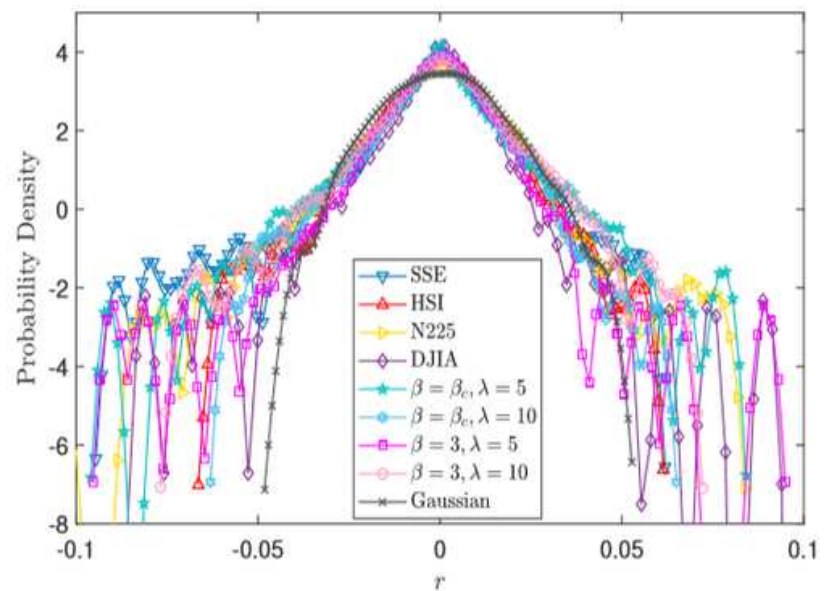

(a)

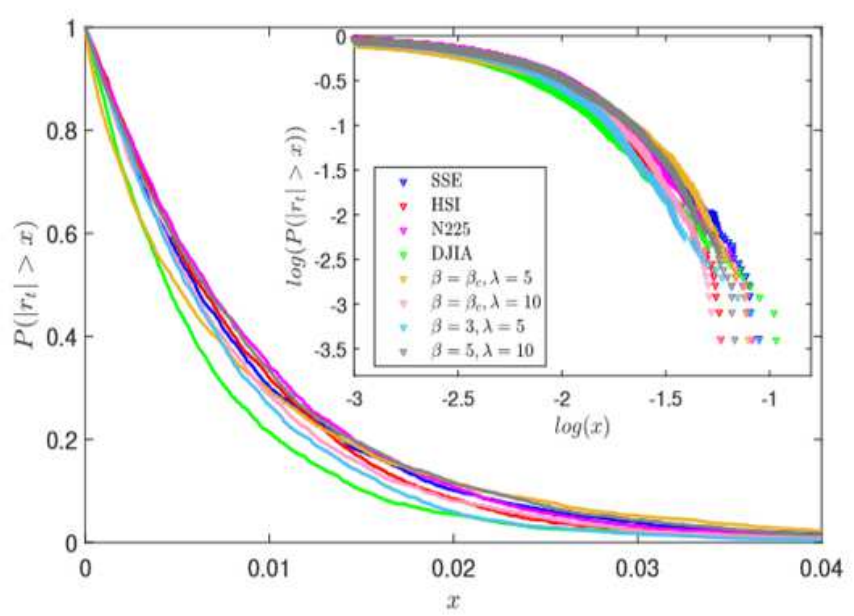

(c)

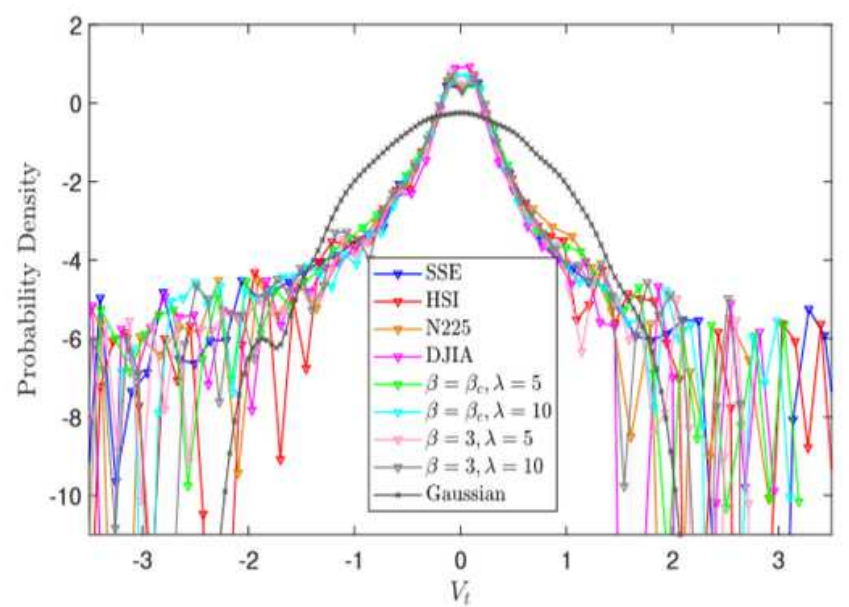

(b)

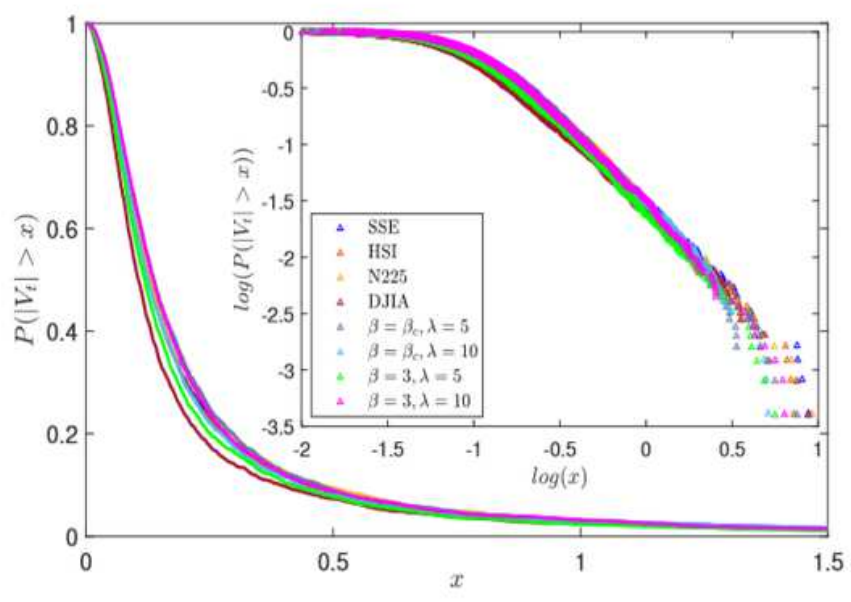

(d)

Figure 4

(a)(b) Logarithmic plots of probability density functions of it and Vt. (c)(d) Plots and log-log plots of cumulative distributions for $|\mathrm{rt}|$ and $|\mathrm{Vt}|$. 


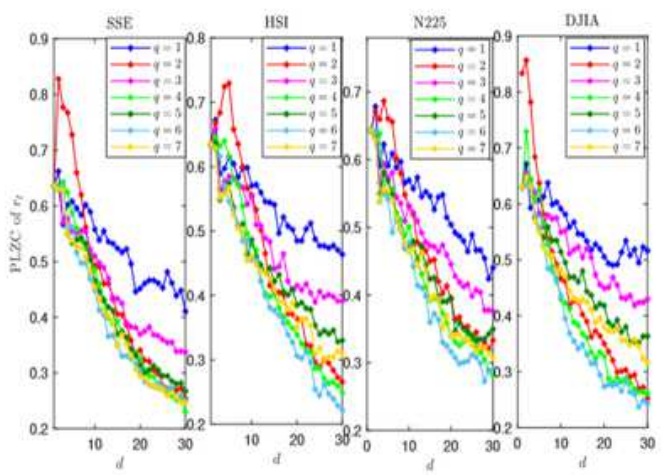

(a)

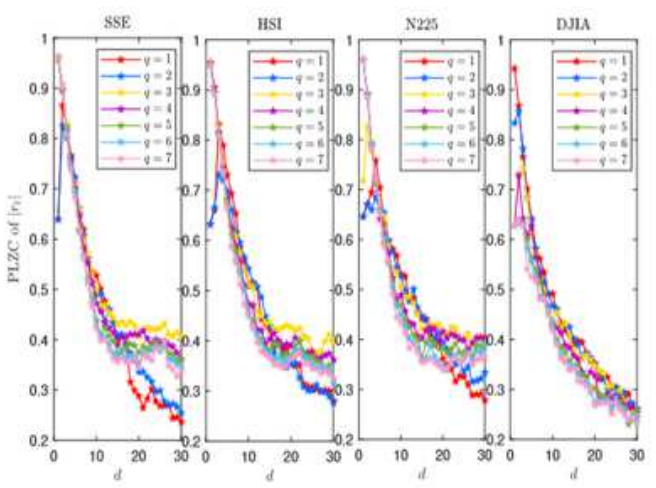

(c)

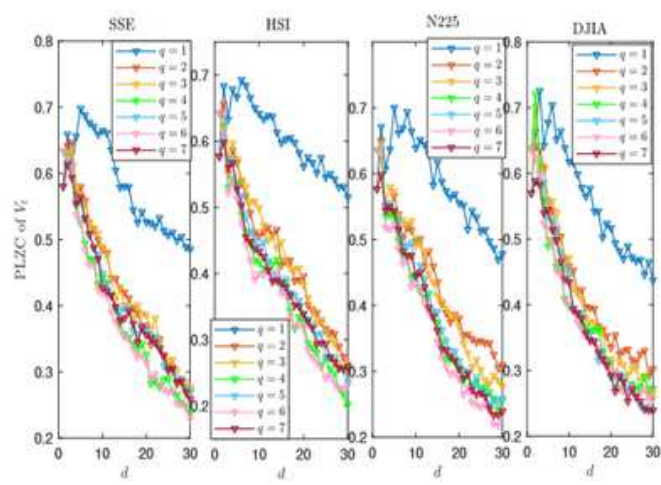

(e)

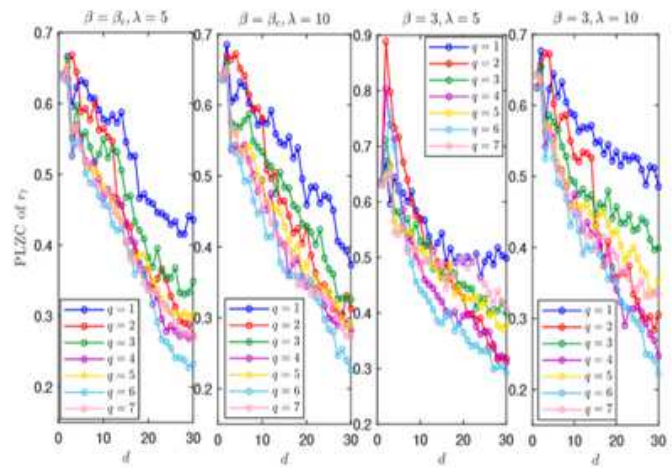

(b)

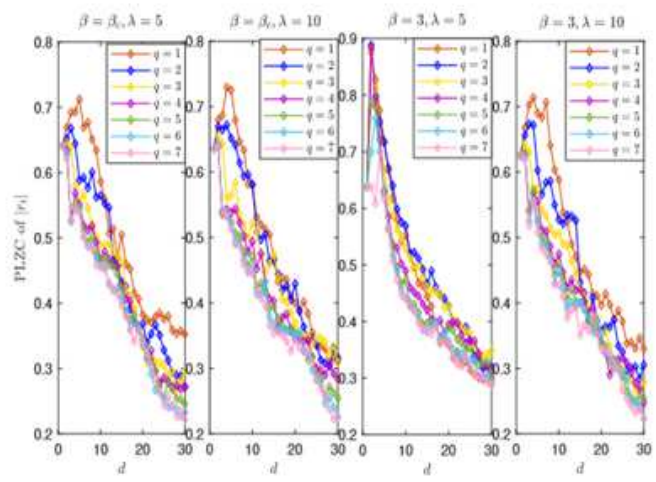

(d)

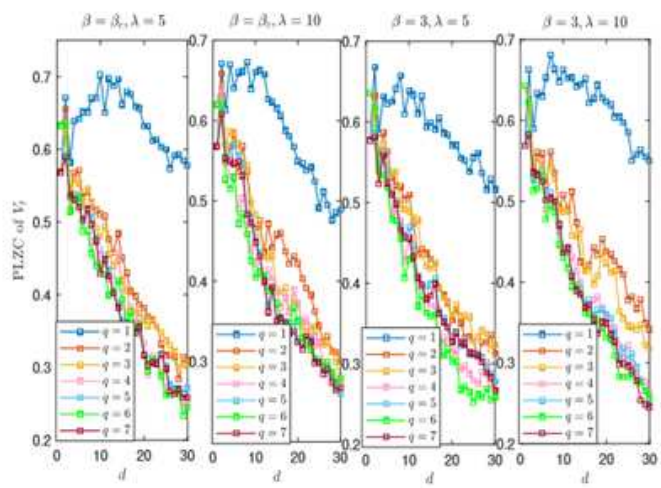

(f)

Figure 5

(a)(b) PLZC plots of rd,q t for real data and simulative data, respectively. (c)(d) PLZC plots of |rt|d,q for real data and simulative data, respectively. (e)(f) PLZC plots of V d,q t for real data and simulative data, respectively. 


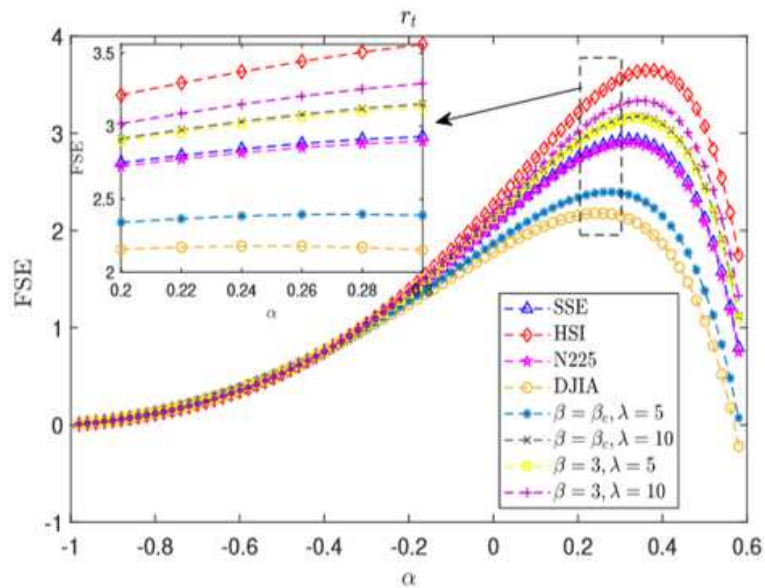

(a)

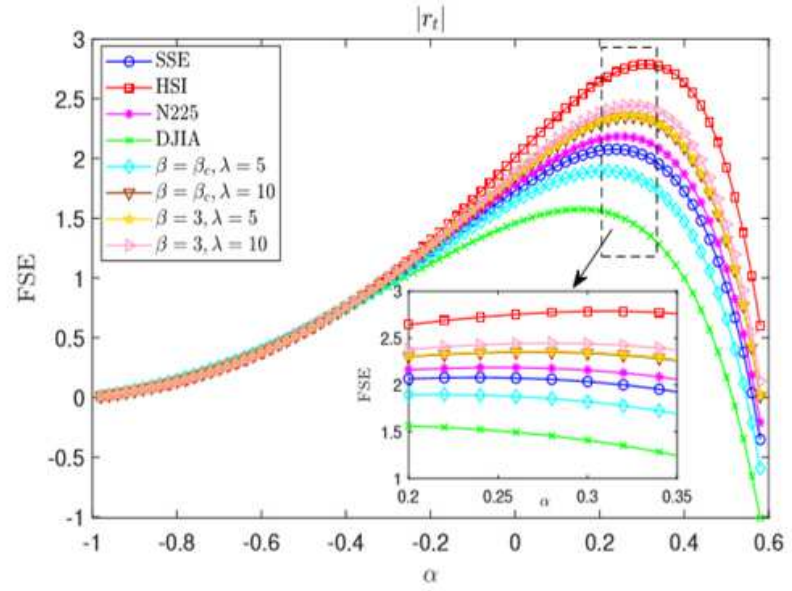

(b)

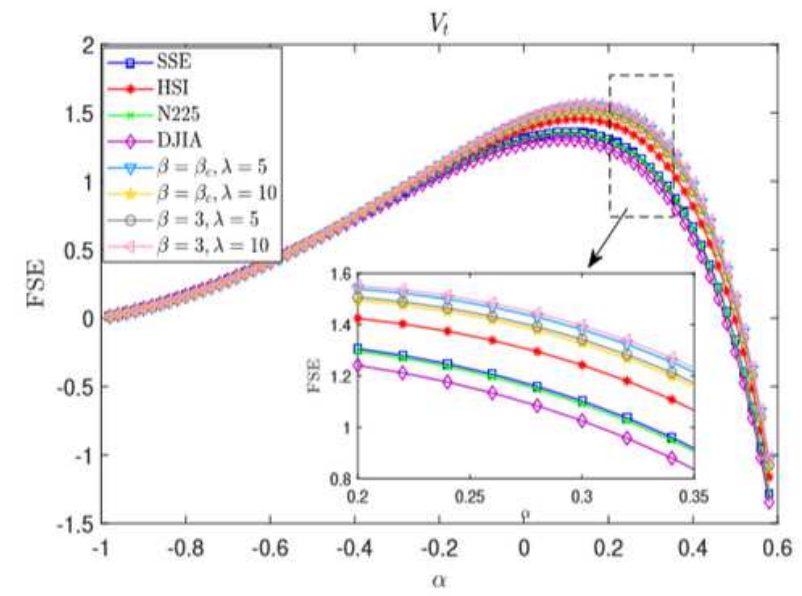

(c)

\section{Figure 6}

(a)(b)(c) FSE curves versus a of volatility sequences $\mathrm{rt}$, |rt|and Vt for real stock data and simulative data. 


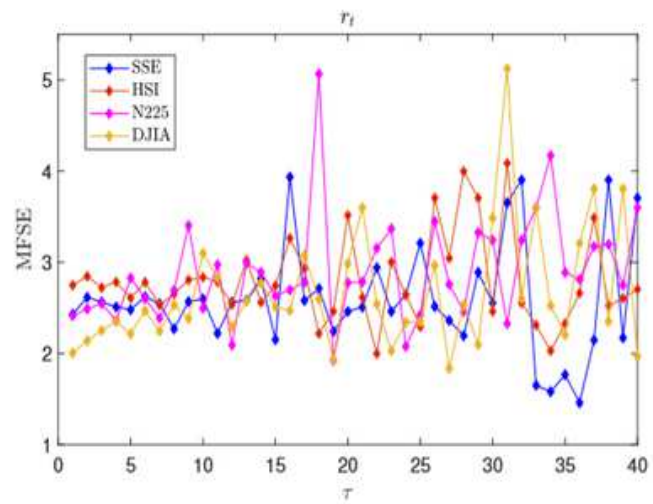

(a)

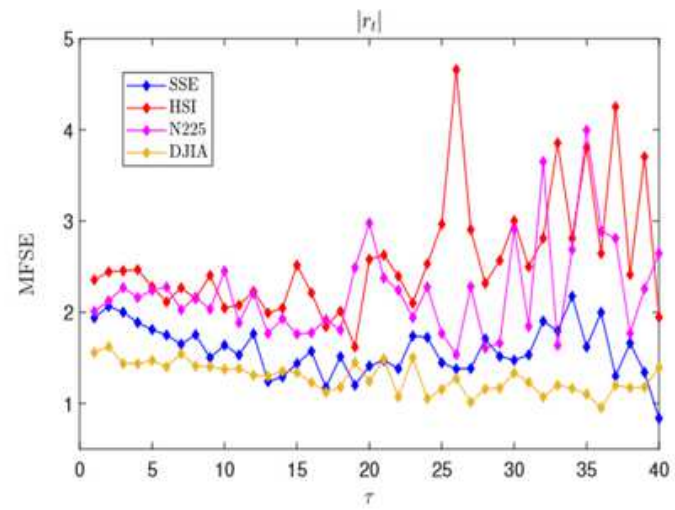

(c)

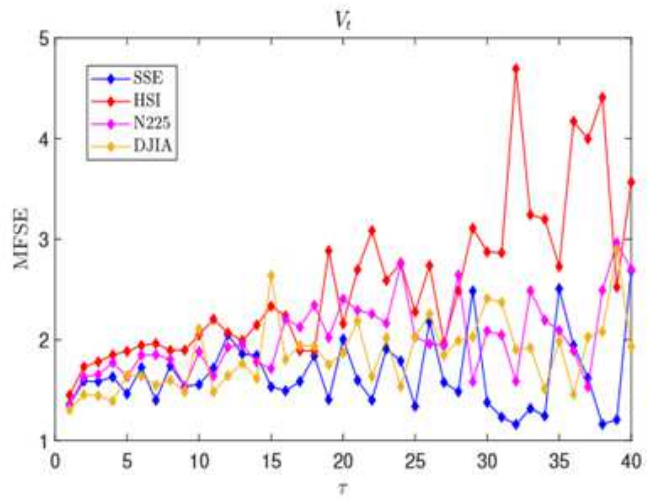

(e)

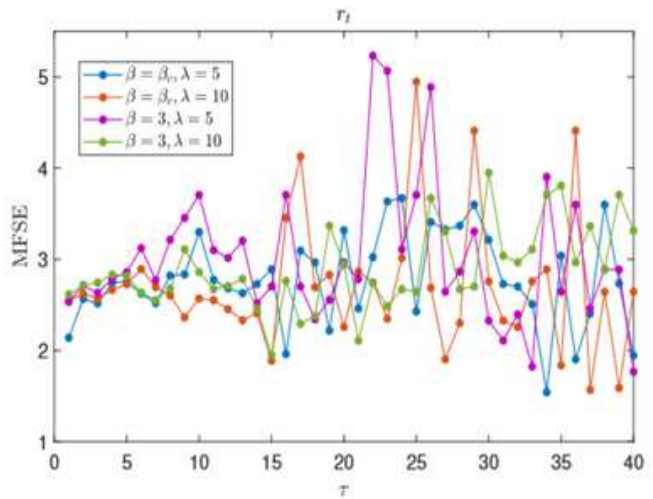

(b)

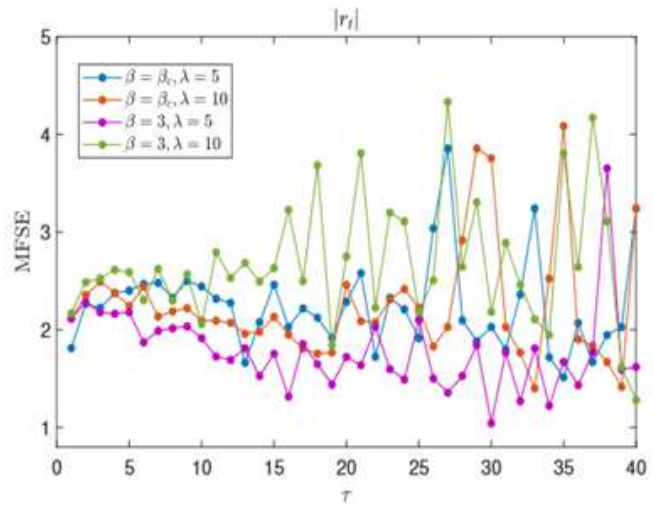

(d)

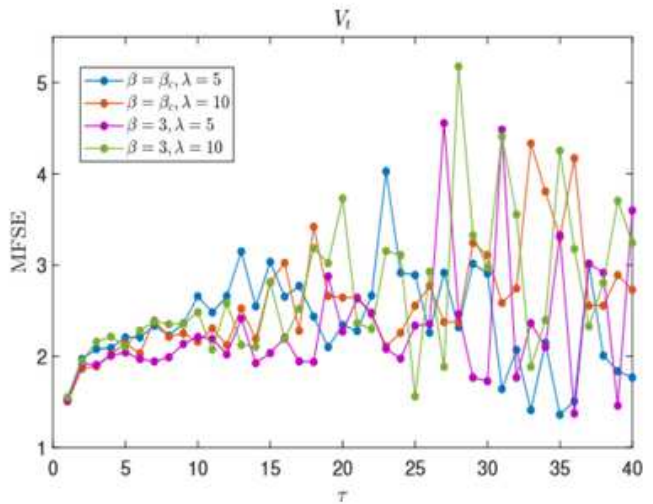

(f)

\section{Figure 7}

(a)(b) MFSE plots of it for real data and simulative data. (c)(d) MFSE plots of |rt| for real data and simulative data. (e)(f) MFSE plots of Vt for real data and simulative data. 